Im vorangegangenen Kapitel haben wir die vielfältigen Aspekte des Geschäftsprozessmanagements umrissen. Da in der Praxis häufig Modelle für die Beschreibung dieser Aspekte zum Einsatz kommen, gehen wir in den folgenden Abschnitten zunächst näher auf die Aufgaben und Eigenschaften von Modellen und Modellbildung ein. Danach stellen wir Beispiele von Modellen aus verschiedenen Fachgebieten vor, welche sich nach unserer Erfahrung in zahlreichen Geschäftsprozessmanagementprojekten als hilfreich erwiesen haben. Die Darstellung erhebt keinen Anspruch auf Vollständigkeit, kann jedoch dem Leser als Orientierung dienen, wenn er selbst Beschreibungsmodelle für sein individuelles Projekt auswählen muss.

\title{
2.1 Modell und Wirklichkeit
}

„Man muss die Welt nicht verstehen, man muss sich nur darin zurecht finden“. Im Internet wird dieser Satz Albert Einstein zugeschrieben. Wer versteht schon, was in der Welt alles geschieht? Wer weiß schon, wie sie funktioniert? Daher sollten wir uns um unsere Welt kümmern, um den Teil der Welt, der uns momentan wichtig ist. Wir sollten erkennen, dass wir uns unsere Welt täglich erschaffen bzw. konstruieren. Ein betrachteter Ausschnitt ist natürlich bestimmt von unseren subjektiven Interessen.

Wir entscheiden, welchen Ausschnitt der Welt wir betrachten wollen, und welche Aspekte uns darin wichtig erscheinen. Wir identifizieren dabei die für uns wesentlichen Artefakte und die für uns wesentlichen Beziehungen zwischen ihnen. Eine solche Abstraktion eines Ausschnitts der Wirklichkeit wird als Modell bezeichnet. Es kann auch sein, dass der betrachtete Ausschnitt der Wirklichkeit bereits ein Modell ist. Damit können Teile eines bereits existierenden Modells näher betrachtet werden. Dies wäre dann ein Modell eines Modells. Dieses Hochschrauben kann beliebig fortgesetzt werden. 


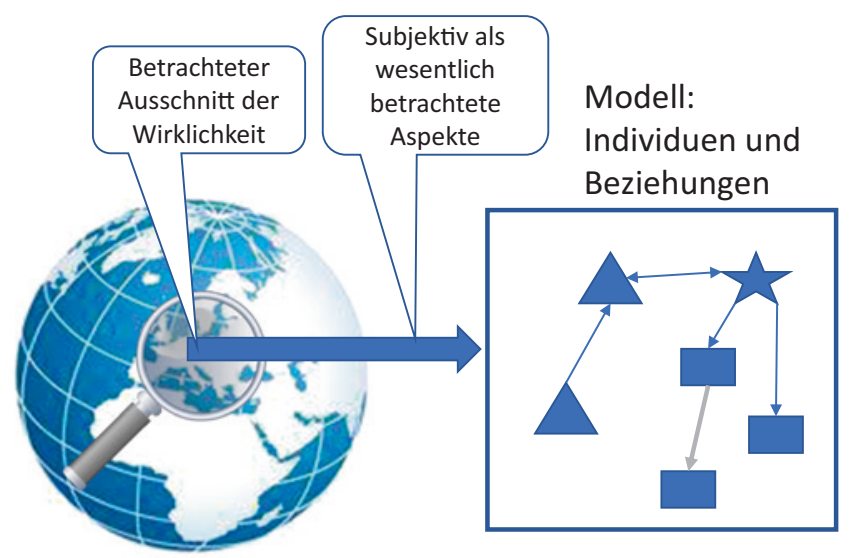

Abb. 2.1 Modellbildung

Jede Person hat eine eigene, subjektive Sicht auf die Welt oder einen Ausschnitt der Welt. Verschiedene Menschen betrachten den gleichen Ausschnitt der Wirklichkeit, kommen allerdings zu unterschiedlichen Modellen, da sie unterschiedliche Schwerpunkte setzen (vgl. Abb. 2.1).

Da Modelle nicht alle Aspekte der zugeordneten Wirklichkeit abdecken, gibt es durchaus Vorfälle in der Wirklichkeit, die durch ein Modell nicht abgedeckt sind und in ihm nicht nachvollziehbar sind. Diese nicht in einem Modell erklärbaren Phänomene bilden dessen Grenzen. Der Erschaffer eines Modells kann nun entscheiden, dass er dieses anpasst, weil er die bisher nicht abgedeckten Phänomene auch mit einbeziehen möchte, oder er legt fest, dass er diese nicht weiter betrachten möchte. Jedes Modell ist und bleibt eine Vereinfachung der Wirklichkeit, weshalb es immer Phänomene geben wird, die von einem Modell nicht erfasst werden.

Jeder Anwender muss entscheiden, ob das von ihm verwendete Modell für seine Versuche, sich in der Welt zurecht zu finden, ausreicht. Möchte man mit einem Modell alle Aspekte der Wirklichkeit abdecken, erreicht es die Komplexität der Wirklichkeit. Dann versteht man das Modell nicht mehr, so, wie man vorher die Wirklichkeit nicht verstanden hat. Der Sinn eines Modells, nämlich die Wirklichkeit besser begreifbar und handhabbar zu machen, wird nicht mehr erreicht.

Das Kreieren eines Modells und seine Verwendung sind subjektive Tätigkeiten, d. h. der Ersteller wählt die Eigenschaften der Wirklichkeitsabbildung nach seinen Vorstellungen aus. Allerdings ist es üblich, dass sich Gruppen von handelnden Personen, in weiterer Folge als Subjekte oder Akteure bezeichnet, auf ein Modell zur Betrachtung der Wirklichkeit einigen. Viele wissenschaftliche Schulen basieren auf solchen gemeinsamen Modellen der involvierten Forscher. 
Modellbildung ist eine wesentliche Tätigkeit in allen Wissenschaften, seien es Philosophie, Soziologie, Physik, Chemie, alle Ingenieurswissenschaften, Ökonomie usw. Die jeweiligen Modelle haben dabei verschiedene Aufgaben: Entweder bilden sie den betrachteten Ausschnitt ab, wie in den Naturwissenschaften üblich, oder sie dienen dazu, bestimmte notwendige Änderungen an dem betrachteten Ausschnitt der Wirklichkeit auszuprobieren (Simulationsmodell). Dies ist beispielsweise wichtig, um keine Menschenleben zu gefährden. Die Sicherheitsmodelle von Autos werden vor deren Serienproduktion in entsprechenden Tests auf ihre Eigenschaften hin überprüft. Dies geschieht nicht mit Menschen, sondern Modellen von Menschen, sogenannten Dummies. Dabei werden zwei Modelle miteinander kombiniert: Das Automodell, das die entsprechenden Sicherheitskonzepte umsetzt, und das Menschenmodell (Dummy), um die Verletzungsrisiken zu untersuchen.

Der in einem Modell erfasste Sachverhalt kann nun so lange angepasst werden, bis bei den mit dem Modell betrachteten Phänomenen das gewünschte Ergebnis erreicht wird. Beispielsweise werden dem Sicherheitsmodell eines Autos weitere Sicherheitskonzepte hinzugefügt, bis die gewünschte Reduktion des Verletzungsrisikos erreicht wird. Solche Modelle sind dann keine Abbilder der Wirklichkeit mehr, sondern stellen eine gewünschte Wirklichkeit dar. Die gewünschten Eigenschaften werden dann in die Wirklichkeit zurück transferiert, indem man beispielsweise die im Modell überprüften Sicherheitskonzepte in die Serienautos einbaut.

Ziel bei der Modellbildung ist immer, dass wir uns in der Welt zurechtfinden, oder gefahrlos ausprobieren können, wie eine entsprechende Änderung der Wirklichkeit sich auswirken würde. $\mathrm{Zu}$ verstehen, was die Welt im Innersten zusammenhält, wird uns wie es aussieht auf absehbare Zeit damit nicht gelingen. Das entsprechende Modell wäre dann die Welt, die es nicht gibt [1].

\subsection{Eigenschaften von Modellen}

Modelle dienen sowohl der abstrakten Darstellung der betrachteten Wirklichkeit im Sinne einer Erkenntnisfunktion als auch der Gestaltung der betrachteten Realität im Sinne eines Rückschlusses. Wie bereits angesprochen wird ein Modell so verändert, dass es einer gewünschten Wirklichkeit entspricht und dann als Bauplan für eine entsprechende Umgestaltung der Realität verwendet. In Naturwissenschaften wie der Physik haben Modelle überwiegend eine Erkenntnisfunktion, während sie in den Ingenieurwissenschaften und der Betriebswirtschaft die Gestaltung der Wirklichkeit unterstützen sollen [2].

Bei der Präzisierung des bisher verwendeten Modellbegriffs lehnen wir uns an Herbert Stachowiak an, der in seiner Modelltheorie die Merkmale und die daraus 
abgeleiteten Eigenschaften von Modellen genauer untersucht hat [3]. Demnach sind Modelle durch mindestens drei Merkmale gekennzeichnet [3]:

\section{Abbildung}

Ein Modell ist stets ein Modell von etwas. Es kann die Abbildung oder Repräsentation eines natürlichen oder künstlichen Originals sein, wobei dieses Original wiederum selbst ein Modell sein kann. Die Originale können auf natürlichem Wege entstanden, technisch produziert oder sonst wie einfach gegeben sein. Modelle können sehr unterschiedlich beschrieben bzw. repräsentiert werden:

- Mentale Modelle: Vorstellung im Kopf

- Verbale Modelle: Natürlich-sprachliche Beschreibung

- Grafische Modelle: Technische Zeichnungen oder sonstige Bilder

- Materielle Modelle: Modelle von Gebäuden

- Formale Modelle: Mathematische Modelle, Computerprogramme usw.

Ein Modell und das Original bilden eine Klasse von Attributen. Unter Attributen versteht man Merkmale und Eigenschaften von Individuen, Relationen zwischen Individuen, Eigenschaften von Eigenschaften, Eigenschaften von Relationen usw. Stachowiak überlässt dem modellierenden Subjekt, was ein Individuum ist. Er sieht Individuen als Attribute nullter Stufe. Mengen von Attributen können zu Klassen zusammengefasst werden und bilden dann Attribute der ersten Stufe. Diese Klassen können wieder zu einer nächsten Attributstufe zusammengefasst werden usw.

\section{Verkürzung}

Ein Modell erfasst im Allgemeinen nicht alle Attribute des betrachteten Originals, sondern nur diejenigen, die dem Modellschaffern bzw. Modellnutzern als relevant erscheinen.

Nicht alle Attribute des Originals werden von einem Modell erfasst. Bereits mit diesem Merkmal ist im weiteren Sinne eine pragmatische Betrachtungsdimension eingeführt worden. Im ,weiteren Sinne“ bedeutet hier, dass noch nicht spezifische pragmatisch-operationale Gesichtspunkte betrachtet werden, nach denen die Attributklassen, die in ein Modell eingehen sollen, selektiert werden. Diese erste Auswahl der Attribute ist intuitiv und willkürlich. Im engeren Sinn pragmatisch ist die Verkürzung erst dann, wenn die Absichten und operationalen Zielsetzungen des Modellerstellers bzw. Modellbenutzers die Auswahl der modellrelevanten Attribute beeinflussen. Diese Anpassungen an den beabsichtigten praktischen Gebrauch erfolgt erst im nächsten Schritt.

3. Pragmatismus

Nach der intuitiven Auswahl der Attribute wird überprüft, ob damit der beabsichtigte Zweck erreicht wird. Modelle sind ihren Originalen nicht eindeutig zugeordnet. Sie erfüllen eine Ersetzungsfunktion

- für bestimmte erkennende bzw. handelnde modellbenutzende Subjekte (für wen?). Modelle sind nicht nur Modelle von etwas, sie sind auch Modelle für jemanden. Dieser Jemand kann ein Mensch oder auch ein künstlicher Modellbenutzer wie 
z. B. ein Computerprogramm sein. Für einen Modellierer können Modelle dazu dienen, sich in der Welt zurecht zu finden, d. h. der Modellierer ist gleichzeitig auch Nutzer des Modells. Modellierer und Modellnutzer können aber auch zwei unterschiedliche Subjekte sein.

- innerhalb bestimmter Zeitintervalle (wann?)

Modelle erfüllen auch eine Funktion über die Zeit, d. h. ihre Nutzung ist auf einen bestimmten Zeitpunkt oder auf ein definiertes Zeitintervall bezogen. Innerhalb dieser Zeit können sich die betrachtete Wirklichkeit oder die Vorstellungen des Modellierers oder Modellanwenders derart verändert haben, dass zusätzliche Attribute in ein Modell einfließen sollen

- unter Einschränkung auf bestimmte gedankliche oder tatsächliche Operationen (wozu?).

Modelle werden zu einem bestimmten Zweck geschaffen, sei es, damit ein bestimmter Ausschnitt der Wirklichkeit besser verstanden werden kann, oder, um einen Bauplan für den Umbau der Wirklichkeit zu bekommen.

Bei der Erstellung eines Modells stecken Modellierende immer in einem gewissen Dilemma. Zum einen soll das Modell in ausreichendem Maße die gewünschten Aspekte der Wirklichkeit wiedergeben, wobei nicht klar definiert ist, was ausreichend ist; Zum anderen soll das Modell nicht zu komplex sein, damit es noch handhabbar bleibt. Dieser Zielkonflikt führt dazu, dass die meisten Modelle iterativ weiterentwickelt werden, bis sie wegen der steigenden Komplexität das Ende ihres Lebenszyklus erreichen, da sie nicht mehr handhabbar sind.

In den folgenden Abschnitten stellen wir Beispiele für Modelle vor, die Aspekte betrachten, welche explizit oder implizit in Modelle für Geschäftsprozesse einfließen. Wir haben die Beispiele gruppiert in Modelle aus den Sozialwissenschaften, der Betriebswirtschaftslehre, der Wirtschaftsinformatik und der Informatik. Die Einordnung in diese Gruppen ist teilweise nicht überschneidungsfrei, da insbesondere die Wirtschaftsinformatik als Querschnittsdisziplin Sachverhalte grundsätzlich aus mehreren Perspektiven betrachtet.

\subsection{Modelle der Sozialwissenschaften}

Geschäftsprozessmanagement hat mit Menschen und Maschinen zu tun. Es möchte deren Zusammenwirken unter Berücksichtigung von Zusatzanforderungen der technischen sowie der ökonomischen und ökologischen Machbarkeit organisieren. Insbesondere das Zusammenwirken und Zusammenleben der Menschen ist schon Jahrtausende lang Thema der Philosophie. Die Philosophie als die Lehre von den grundlegenden Bestimmungen und Strukturen des Lebens, der Welt und des Wissens versucht, die Welt und die menschliche Existenz zu ergründen, zu deuten und zu verstehen. Die ursprüngliche Bedeutung von Philosophie war die Lehre vom guten Leben. 
In diesem Sinn ist Philosophie der Versuch, das umfassende Modell unserer Welt zu kreieren was bisher noch nicht gelungen ist. Gesellschaftsphilosophen reduzieren den Blick und versuchen ein Modell der Gesellschaft als einen Ausschnitt der Wirklichkeit zu schaffen und damit deren Sinn und Wesen besser zu verstehen. Insbesondere beleuchten Gesellschaftsphilosophien das Verhältnis zwischen dem einzelnen Menschen und der Gemeinschaft sowie die Strukturen des Zusammenlebens. Sie gelten deshalb auch als Philosophievarianten, welche die Soziologie berühren. Sie sollen Soziologen dabei helfen, gesellschaftliche Vorgänge zu analysieren und Organisationsentwickler bei ihrer Arbeit unterstützen, und den ,normalen“ Menschen helfen, sich in der Welt zurecht zu finden. Es gibt zahlreiche Organisationstheorien, die in ihren Modellen auf unterschiedliche Aspekte fokussieren [4-7]. Die Frage, welche Organisationstheorie die Beste ist kann nicht beantwortet werden. Organisationstheorien repräsentieren Modelle und damit (nach Stachowiak) die zwar begründete, aber subjektive Sicht des Modellierers. Organisationstheorien setzen höchst unterschiedliche Schwerpunkte bei der Analyse von Organisationen und verfolgen unterschiedliche Zielsetzungen. Es gibt zu den jeweiligen Organisationstheorien empirische Untersuchungen, die Ergebnisse zugunsten oder Ungunsten einer Theorie liefern. Allerdings sind die verwendeten Untersuchungs- sind Analysemethoden umstritten [7].

Organisationstheorien liegen bestimmte Menschenbilder zugrunde, und die Ausgestaltung des Geschäftsprozessmanagements wird stark von dem in einer Organisation herrschenden Menschenbild geprägt. Deshalb stellen wir mit dem Taylorismus, der Habermas'schen Theorie des kommunikativen Handelns, und den sozialen Systemen von Luhmann drei Organisationstheorien mit ihren Menschenbildern in den folgenden Abschnitten vor ${ }^{1}$.

\subsubsection{Taylorismus und Fordismus}

Der Taylorismus führte das Experiment in die Managementlehre und -praxis ein, und zählt zu den Klassikern der Organisationslehre. Mit dem sogenannten Scientific Management erhielten Organisationen ein Instrument zu ihrer effizienten Gestaltung. Ein wesentliches Kennzeichen ist die Trennung zwischen planender Kopfarbeit und ausführender Handarbeit. Nach dem Tayloristischen Menschenbild sind Arbeiter dumm und faul und müssen deshalb strengen Regeln unterworfen werden. Diese Sicht beeinflusst auch heute noch oft implizit das Verhalten von Führungskräften.

Für Frederik Winslow Taylor waren die wichtigsten Ziele die Vervollkommnung der Produktionsmittel und Arbeitsverfahren, straffere Organisation und Zeitordnung des Arbeitsablaufes im Betrieb, sowie eine Neuordnung des Entlohnungssystems. Ein Kernelement des Taylorismus ist die Gestaltung von Arbeitsprozessen auf der Grundlage

${ }^{1}$ Ein Überblick über Organisationstheorien findet sich in [7]. 
von Zeit- und Bewegungsstudien. Eine Zerlegung des Produktionsprozesses in kleinste Arbeitsschritte, eine Entlastung der Arbeiter von geistigen Tätigkeiten, sowie eine Änderung des Lohnsystems sollen zu einer optimalen Nutzung der vorhandenen Leistungspotenziale führen.

Ziel ist die Steigerung der Produktivität menschlicher Arbeit. Dies geschieht durch die Teilung der Arbeit in kleinste Einheiten, zu deren Bewältigung keine, oder nur geringe Denkvorgänge zu leisten, und die aufgrund des geringen Umfangs bzw. Arbeitsinhalts schnell und repetitiv zu wiederholen sind.

Dem Taylorismus liegen folgende Kernprinzipien zugrunde:

- Arbeitsplanung

Die Planung der Arbeit wird von anderen Personen durchgeführt als deren Ausführung (Trennung von Hand- und Kopfarbeit). Damit wollte Taylor die Drückebergerei, die er den Arbeitern unterstellte, umgehen. Durch Zeit und Bewegungsstudien, die von den Kopfarbeitern ausgeführt wurden, sollte der geringste Bewegungs- und Zeitaufwand für einen Arbeitsschritt ermittelt werden.

- Leistungslohn

Aus diesen Zeit- und Bewegungsstudien ergab sich auch, was die Arbeiter in einer gewissen Zeit zu leisten hatten. Ein Bonus oder eine Prämie sorgten dafür, dass sich die als dumm und faul eingestuften Arbeiter auch tatsächlich bemühten, die vorgegebenen Leistungsdaten zu erreichen.

- Auswahl der am besten geeigneten Arbeiter

Durch eine entsprechende Auslese galt es einen erstklassigen Arbeiterstamm aufzubauen. Es wurden entsprechende Tests entwickelt und eingesetzt, um besonders fingerfertige und flinke Arbeiter zu identifizieren.

- Versöhnung zwischen Arbeitern und Management

Taylor glaubte, dass durch das von ihm entwickelte System die Produktivität so gesteigert werden kann, dass der Streit um die Verteilung des Gewinns zur Nebensache werden würde. Dadurch sollte der Konflikt zwischen Arbeitgebern und Arbeitnehmern aufgelöst werden.

Der Taylorismus betrachtet im Wesentlichen die Strukturierung der Arbeitsschritte, stellt jedoch nicht auf deren Reihenfolge ab. Diesen Aspekt ging Henry Ford an, der mit der Einführung des Fließbands die einzelnen Tätigkeiten koordinierte. Damit waren die Voraussetzungen für die Massenfertigung geschaffen, die das 20. Jahrhundert prägte. Das Fließbandprinzip wurde auch in die Verwaltung übertragen und beeinflusste stark das Geschäftsprozessmanagement. Flussdiagramme sind die Fließbandvorgaben für die Abwicklung von Verwaltungsaufgaben oder die „Produktion“ von Dienstleistungen. 


\subsubsection{Kommunikatives Handeln nach Habermas}

Im Gegensatz zum Taylorismus geht die Theorie des kommunikativen Handelns nach Habermas von einsichtigen Menschen aus, die durch die Kommunikation untereinander zu einem gemeinsamen vernünftigen Handeln kommen. Mit seinem Gesellschaftsmodell erklärt Habermas die Vorgänge in einer Gesellschaft, wie z. B. die Suche nach Wahrheit, nach Gerechtigkeit. Es ist also ein Modell, das alle angeht, denn die Themen Wahrheit und Gerechtigkeit betreffen sämtliche Mitglieder von Gesellschaften. Der zentrale Aspekt im Gesellschaftsmodell von Habermas ist das sogenannte kommunikative Handeln.

„Der Begriff des kommunikativen Handelns schließlich bezieht sich auf die Interaktion von mindestens zwei sprach- und handlungsfähigen Subjekten, die (sei es mit verbalen oder extraverbalen Mitteln) eine interpersonale Beziehung eingehen. Die Aktoren suchen eine Verständigung über die Handlungssituation, um ihre Handlungspläne und damit ihre Handlungen einvernehmlich zu koordinieren“ [8].

Nach Habermas gelingt es durch Kommunikation, dass der einzelne Mensch, der von sich aus nicht vernunftbegabt ist, diesen Mangel überwindet. Die Kommunikation zwischen Menschen wird zum intersubjektiven Handeln und zu einer möglichen Quelle der Vernunft. Kommunikatives Handeln heißt Handeln auf der Grundlage der Verständigung zwischen Menschen.

Habermas möchte damit Soziologen und Politikern ein Modell anbieten, das sie nutzen können, um die Gesellschaft zu analysieren bzw. zu gestalten. Dem einzelnen Menschen kann es dazu dienen, sich trotz der Komplexität heutiger Gesellschaften zurecht zu finden.

\subsubsection{Soziale Systeme nach Luhmann}

Ähnlich wie bei Habermas basiert das Gesellschaftsmodell von Luhmann auf Kommunikation. Die Unterschiede liegen darin, in wieweit Kommunikation und Handeln kombiniert sind. Luhmann lässt ausschließlich Kommunikation als konstituierenden Aspekt für Organisationen zu - Kommunikation findet nicht zwischen Menschen statt, sondern zwischen mindestens zwei informationsverarbeitenden Prozessoren. Damit sieht Luhmann die Kommunikation abstrakter. Nach ihm besteht die Gesellschaft nicht aus Menschen oder Teilen von Menschen. Sonst würde man etwas von der Gesellschaft abschneiden, wenn man etwas vom Menschen abschneidet. Der Körper eines Menschen (als biologisches System) mit einem Bewusstsein (psychisches System) ist zwar in vielen Fällen Voraussetzung für das Funktionieren eines sozialen Systems, also der Kommunikation, aber ein Mensch ist nicht das soziale System selbst. Luhmann macht in seiner Organisationstheorie keinerlei Aussagen zur Natur des Menschen, lässt also das Menschenbild offen. Menschen sind nur insofern Teil der Organisation, als sie miteinander kommunizieren.

Die Kommunikation zwischen den informationsverarbeitenden Prozessoren besteht aus den sogenannten Selektionen der Information, der Mitteilung und des Verstehens 


\begin{tabular}{|c|c|c|}
\hline & \multicolumn{2}{|c|}{$\begin{array}{l}\text { Zwei informationsverarbeitende Prozessoren } \\
\text { In der Regel Personen oder soziale Systeme }\end{array}$} \\
\hline & $\begin{array}{c}\text { Sender } \\
\text { Bei Luhmann: „Alter“ }\end{array}$ & $\begin{array}{c}\text { Empfänger } \\
\text { Bei Luhmann: „Ego“ }\end{array}$ \\
\hline Drei Selektionen: & $\begin{array}{l}\text { 1. Selektion der Information } \\
\text { 2. Selektion der Mitteilung }\end{array}$ & $\begin{array}{l}\text { 3. Selektion der Annahme/ des } \\
\text { Verstehens }\end{array}$ \\
\hline
\end{tabular}

Abb. 2.2 Luhmann'sches Kommunikationsverständnis

(siehe Abb. 2.2). Die ersten beiden Selektionen liegen beim Sender und die dritte beim Empfänger. Die Kommunikation als Stück mit mindestens zwei Akteuren in drei Akten ist eine unteilbare Einheit, nämlich die kleinste Einheit eines sozialen Systems und die elementare Operation der Gesellschaft [9]. Diese Sicht kann als Muster zur Definition der Kommunikation in Geschäftsprozessen dienen, vollkommen unabhängig von einem konkreten Menschenbild.

Sowohl Luhmann als auch Habermas stellen in ihrer Organisationstheorie also die Kommunikation in den Mittelpunkt der Betrachtung. Dass diese beiden bedeutenden Organisationstheoretiker den Kommunikationsaspekt der Organisation so stark betonen und ihre Theorien weitreichend akzeptiert sind, kann als Indiz gelten, Geschäftsprozesse primär kommunikationsorientiert zu betrachten.

\subsubsection{Organisationen}

Komplexe soziale Systeme können in kleinere soziale System gegliedert werden. Diese Struktur eines komplexen sozialen Systems wird als Organisationsstruktur bezeichnet. Nach welchen Kriterien die Aufteilung in kleinere soziale System erfolgt ist subjektiv und hängt von den jeweiligen Absichten ab. Entsprechend der Luhmann'schen Definition eines sozialen Systems kommunizieren die einzelnen sozialen Systeme innerhalb eines komplexeren sozialen Systems. Organisationsstrukturen sind also ein Modell eines komplexeren sozialen Systems.

In Abgrenzung zum weiten Verständnis des Begriffs Organisation bei Luhmann oder Habermas hat sich auch ein engeres Verständnis von Organisationen entwickelt. In der Betriebswirtschaft wird unter Organisation das formale Regelwerk eines arbeitsteiligen Systems verstanden. In der Organisationssoziologie bezeichnet sie eine besondere Form von sozialem Gebilde, die sich von anderen sozialen Gebilden wie beispielsweise Familien, Gruppen, Bewegungen oder Netzwerken unterscheiden lässt. Wesentliche Kennzeichen von Organisationen sind, dass Personen sich ihnen anschließen oder sie verlassen können.

Darüber hinaus besitzen sie einen Zweck auf den hin sie sich ausrichten. Organisationen haben Regelungen zur Arbeitsteilung wie Spezialisierung nach Verrichtung, 
Funktion, Objekten oder Raum bzw. entsprechende Mischformen. Diese Arbeitsteilung erfordert die Koordination der einzelnen Tätigkeiten. Als zentrales Instrument der Koordination gilt in der Organisationslehre die Hierarchie. Die hierarchische Koordination wird ergänzt durch Stäbe, Kommissionen, Arbeitskreise etc. Bei einmaligen oder erstmalig zu lösenden Problemen wird die Hierarchie durch eine Projektorganisation ergänzt.

\subsection{Modelle der Betriebswirtschaft}

Betriebswirtschaftslehre ist die Lehre von den wirtschaftlichen, organisatorischen, technischen sowie finanziellen Abläufen und Strukturen in Unternehmen. Damit ist auch das Geschäftsprozessmanagement ein Teil der Betriebswirtschaft. Denn Geschäftsprozesse dienen dazu die Wirtschaftlichkeit eines Unternehmens zu verbessern, und zwar mit all den zugehörigen Aspekten wie Kundenzufriedenheit, Mitarbeitermotivation, Einbindung von Partnern usw. Für die Strukturierung all dieser Aspekte und zur Analyse ihres Zusammenwirkens hat die Betriebswirtschaft Modelle entwickelt, die bei ihrer Anwendung in das Geschäftsprozessmanagement hineinwirken.

\subsubsection{Geschäftsmodell}

Ein Geschäftsmodell (Business Model) bezieht sich auf das Gesamtkonzept eines Unternehmens. Es repräsentiert modellhaft die Zusammenhänge, wie dieses für seine Kunden Mehrwert erzeugen und damit nachhaltig Erträge erzielen kann. Neben den angebotenen Produkten und Services geht es u. a. um die Struktur des Unternehmens, die Definition der Zielgruppen (Kunden) und deren Ansprache sowie um die Gestaltung der Geschäftsprozesse. Über dieses Verständnis hinaus gibt es für den Begriff des Geschäftsmodells eine Reihe weiterer Definitionen [10].

Ein Geschäftsmodell dient folglich dazu, den Zusammenhang zwischen dem Unternehmen als Handlungssystem und der Wertschöpfung zu verstehen. Es spiegelt wider, wie eine Firma funktioniert und welche Werte sie für bestimmte Zielgruppen erzeugt. Geschäftsmodelle werden im Rahmen einer Unternehmensgründung oder einer Neuausrichtung erstellt. Sie bestehen aus mehreren Teilmodellen, in denen beschrieben wird, welche Ressourcen (Materialien, Informationen usw.) einem Unternehmen als Eingangsgrößen zur Verfügung stehen (müssen), und wie diese Ressourcen bearbeitet und in vermarktungsfähige Produkte oder Dienstleistungen umgeformt werden, die dann zum Kunden transferiert werden, um entsprechende Einnahmen zu erzielen [11].

Geschäftsmodelle können mehreren Stakeholdern dienen. Die Unternehmensführung kann damit das eigene Geschäft besser verstehen, bestehende Stärken und Schwächen sowie Möglichkeiten zur Weiterentwicklung, Transformation und Verbesserung der Wettbewerbsposition erkennen. Für Investoren ist das Geschäftsmodell oft ein wichtiger Aspekt bei Anlageentscheidungen. 
Zur Erstellung von Geschäftsmodellen wurde eine Reihe von Instrumenten entwickelt. Das bekannteste ist der Business Model Canvas von Alexander Osterwalder [12], der in den letzten Jahren große Akzeptanz fand. Wie der Name sagt, geht der Business Model Canvas-Ansatz von einem Plakat aus, auf dem die betrachteten Aspekte des Geschäftsmodells visualisiert werden. Der Canvas liefert ein Raster für neun Geschäftsmodellaspekte, welches mit den konkreten Ausprägungen der Aspekte für das betrachtete Unternehmen gefüllt wird (vgl. Abb. 2.3). Im Zentrum steht das Werteversprechen (Produkt oder Dienstleistung).

Beim Ausfüllen gilt es jeweils eine Reihe von Fragestellungen zu den neun Aspekten zu beantworten. Die folgenden Ausführungen erläutern die Aspekte kurz und geben eine Auswahl wichtiger dazugehöriger Fragen wieder.

1. Kundensegmente, Zielgruppen:

Alle Personen oder Organisationen, für die das betrachtete Unternehmen Werte kreieren will.

$\mathrm{Zu}$ beantwortende Fragen sind $\mathrm{u}$. a.:

- Wer profitiert vom Produkt oder der Dienstleistung?

- Welche Kunden sind besonders wichtig?

2. Werteversprechen, Kundenutzen:

Für jedes Kundensegment gibt es ein eigenes Werteversprechen, den Kundennutzen. Dies ist eine auf die Bedürfnisse des jeweiligen Segments abgestimmte Kombination aus Produkt und Dienstleistung.

$\mathrm{Zu}$ beantwortende Fragen sind u. a.:

- Welchen Nutzen bzw. Wert hat das Angebot für die Kunden?

- Welche Kundenprobleme werden mit den angebotenen Produkten und/oder Dienstleistungen gelöst?

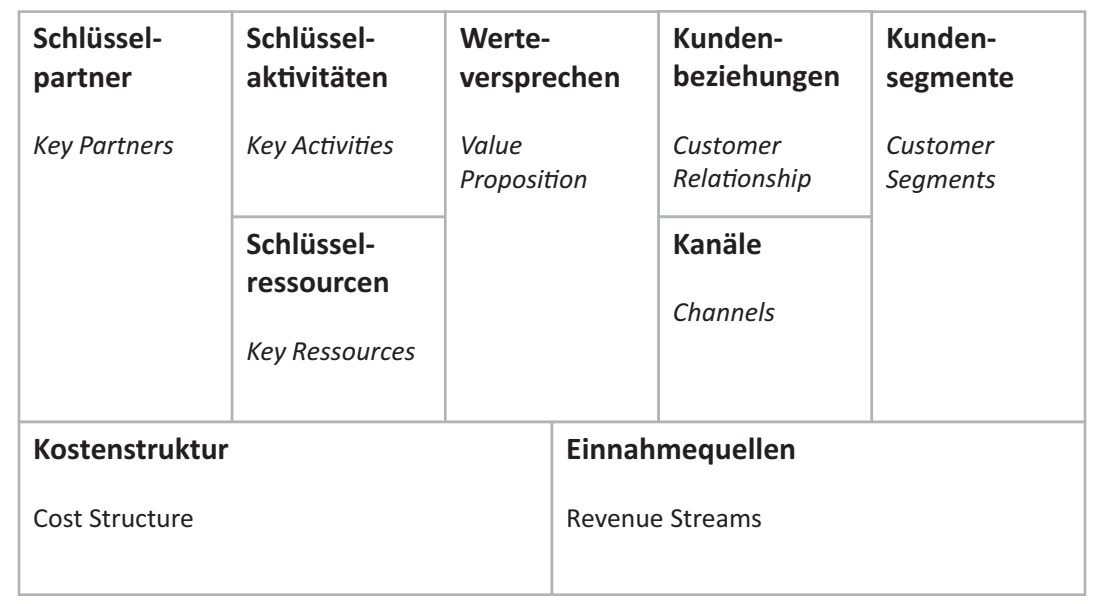

Abb. 2.3 Schema des Business Model Canvas 
3. Kanäle, Vertriebswege:

Dieser Faktor steht für die einzelnen Kanäle, über die mit den Kunden kommuniziert wird und ihnen die versprochenen Werte übermittelt werden. Die Vertriebskanäle bestimmen, wie die Interaktion mit den Kunden abläuft. Kommunikation, Distribution und Verkaufsstellen bilden Schnittstellen eines Unternehmens zu seinen Kunden. Die Wahrnehmung des Kunden an diesen Berührungspunkten ist dabei zentral und bestimmt den Eindruck, den ein Kunde von einem Unternehmen hat.

$\mathrm{Zu}$ beantwortende Fragen sind u. a.:

- Wie erfahren Kunden von den angebotenen Produkten und Dienstleistungen?

- Wie gelangen die Produkte/Dienstleistungen zum Kunden?

4. Kundenbeziehungen:

Hier wird beschrieben, welche Form des Umgangs mit den Kunden gepflegt wird.

Jedes Unternehmen sollte sich darüber Gedanken machen, welche Arten von Kundenbeziehungen es mit den verschiedenen Zielgruppen eingehen möchte. Dabei hängt die Gestaltung der Kundenbeziehungen nicht nur von der jeweiligen Zielgruppe ab, sondern auch von den damit verbundenen Zielen des Unternehmens (Neukundengewinnung, Bestandskundenpflege etc.).

$\mathrm{Zu}$ beantwortende Fragen sind u. a.:

- Welche Art von Beziehung erwarten die einzelnen Kundengruppen?

- Wie wird die Beziehung zu den Kunden organisiert?

- Was kostet die Pflege des Kundenkontakts und was bringt dieser Kunde?

5. Einnahmequellen, Erlösmodelle:

Das Unternehmen schafft mit seinem Angebot einen Mehrwert. Die zentrale Frage ist, wie viel der Kunde bereit ist, dafür zu bezahlen. Das Unternehmen trifft eine Entscheidung bezüglich der Preismodelle und der Preisstrategie (Einmalzahlung, Abonnement etc.).

$\mathrm{Zu}$ beantwortende Fragen sind u. a.:

- Wofür und wie viel sind Kunden wirklich bereit für das Angebot zu zahlen?

- Wie viel trägt jede der einzelnen Umsatzquellen zum Gesamtumsatz bei?

- Wie würden die Kunden gerne zahlen?

6. Schlüsselressourcen:

Zur Erstellung des Angebots sind in jeder Unternehmung bestimmte Ressourcen erforderlich. Diese können sich im eigenen Besitz befinden, aber auch gemietet oder von strategischen Partnern zur Verfügung gestellt werden.

$\mathrm{Zu}$ beantwortende Fragen sind $\mathrm{u}$. a.:

- Welche physischen Ressourcen (Räumlichkeiten, Produktionsmaschinen) werden benötigt, um ein Produkt oder einen Service erstellen und anbieten zu können?

- Welche intellektuellen Ressourcen (Wissen, Patente, Partnerschaften, Kundenstamm) werden benötigt?

- Welche personellen Ressourcen (Team) werden benötigt?

- Welche finanziellen Ressourcen (verfügbares Kapital, Sicherheiten) werden benötigt?

- Wie können die nötigen Ressourcen beschafft und vorgehalten werden? 
7. Schlüsselaktivitäten:

Schlüsselaktivitäten sind die für die Leistungserstellung und -verwertung nötigen Tätigkeiten, z. B. Produktion, Vertrieb.

$\mathrm{Zu}$ beantwortende Fragen sind u. a.:

- Welche Schlüsselaktivitäten müssen ausgeführt werden, um ein Produkt oder einen Service anbieten und damit den Kundennutzen realisieren zu können?

- Welche Aktivitäten für welche Vertriebskanäle?

- Welche Aktivitäten für welche Kundenbeziehungen?

8. Schlüsselpartner:

Schlüsselpartner sind Geschäftspartner, die wichtige Ressourcen für die Realisierung des Geschäftsmodells bereitstellen.

Mit ihnen gehen Unternehmen oft strategische Allianzen ein. Beispiele sind Lieferanten, Service Provider o. Ä.

$\mathrm{Zu}$ beantwortende Fragen sind $\mathrm{u}$. a.:

- Wer sind Schlüsselpartner und was tun diese für das Unternehmen?

- Welche Schlüsselressourcen werden von welchen Partnern zur Verfügung gestellt?

9. Kostenstruktur:

Die Kostenstruktur gibt Aufschluss über die wichtigsten Kostenfaktoren eines Geschäftsmodells.

$\mathrm{Zu}$ beantwortende Fragen sind $\mathrm{u}$. a.:

- Was sind die größten und wichtigsten Kostenfaktoren in dem Geschäftsmodell?

- Welche Schlüsselressourcen/Schlüsselaktivitäten sind die teuersten?

Aus den einzelnen Feldern eines Geschäftsmodells lassen sich Kennzahlen und die zugehörigen Zielwerte für Geschäftsprozesse ableiten. Umgekehrt können die Kennzahlen und Zielwerte den Entwurf der Prozesse beeinflussen. Liegt der Fokus auf niedrigen Preisen, werden Prozesse anders aussehen, als wenn das Geschäftsmodell eher auf hoher Qualität ausgerichtet ist.

\subsubsection{Balanced Scorecard}

Die Balanced Scorecard (BSC) wurde Anfang der 1990er-Jahre von Kaplan und Norton vorgestellt [13]. Sie ist ein Bindeglied zwischen dem Geschäftsmodell, der Strategiefindung und deren Umsetzung. Unter Strategie werden in der Wirtschaft klassisch die (meist langfristig) geplanten Verhaltensweisen der Unternehmen zur Erreichung ihrer Ziele verstanden.

Eine BSC beginnt bei der Vision und Strategie eines Unternehmens und definiert auf dieser Basis die kritischen Erfolgsfaktoren (KEF) mithilfe von Kennzahlen und dazugehörigen Zielwerten. Die Vision eines Unternehmens beschreibt das langfristige, ambitionierte Ziel, das eine Organisation bzw. Unternehmen anstrebt. Typische Visionen sind Formulierungen wie „Wir wollen Marktführer in unserem Marktsegment werden“ oder „Wir wollen das profitabelste Unternehmen in unserem Marktsegment werden“. 
Die Kennzahlen fördern die Zielsetzung und Leistungsfähigkeit in kritischen Bereichen der Strategie, um die Vision zu erreichen. Die BSC ist daher ein Management-System, das aus der Vision als Teil des Geschäftsmodells und der Strategie zur Umsetzung des Geschäftsmodells abgeleitet wird, und die wichtigsten Aspekte des Unternehmens widerspiegelt. Das BSC-Konzept unterstützt strategische Planung und Implementierung durch eine Bündelung der Maßnahmen aller Einheiten eines Unternehmens auf der Basis eines gemeinsamen Verständnisses seiner Ziele und durch einen leichteren Zugang zur Bewertung und Fortschreibung der Strategie.

Da traditionelles, rein auf finanzielle Kennzahlen aufgebautes Management den Anforderungen von Unternehmen im Informationszeitalter an effektive Planungswerkzeuge nicht mehr genügt, haben Kaplan und Norton für die BSC vier Perspektiven eingeführt, aus deren Blickwinkel die Aktivitäten eines Unternehmens umfassend bewertet werden können. Für jede Perspektive werden Ziele, Kennzahlen, Vorgaben und Maßnahmen definiert (vgl. Abb. 2.4).

\subsubsection{Total Quality Management und EFQM}

Der Begriff des Total Quality Management (TQM) steht für die Optimierung der Qualität von Produkten und Dienstleistungen eines Unternehmens in allen Funktionsbereichen und auf allen Ebenen durch Mitwirkung aller Mitarbeiter. Optimierung der Qualität bedeutet dabei, weder mit gegebenem Aufwand das höchste Qualitätsniveau zu erreichen, noch die Qualität ohne Rücksicht auf Kosten zu steigern. Vielmehr geht es

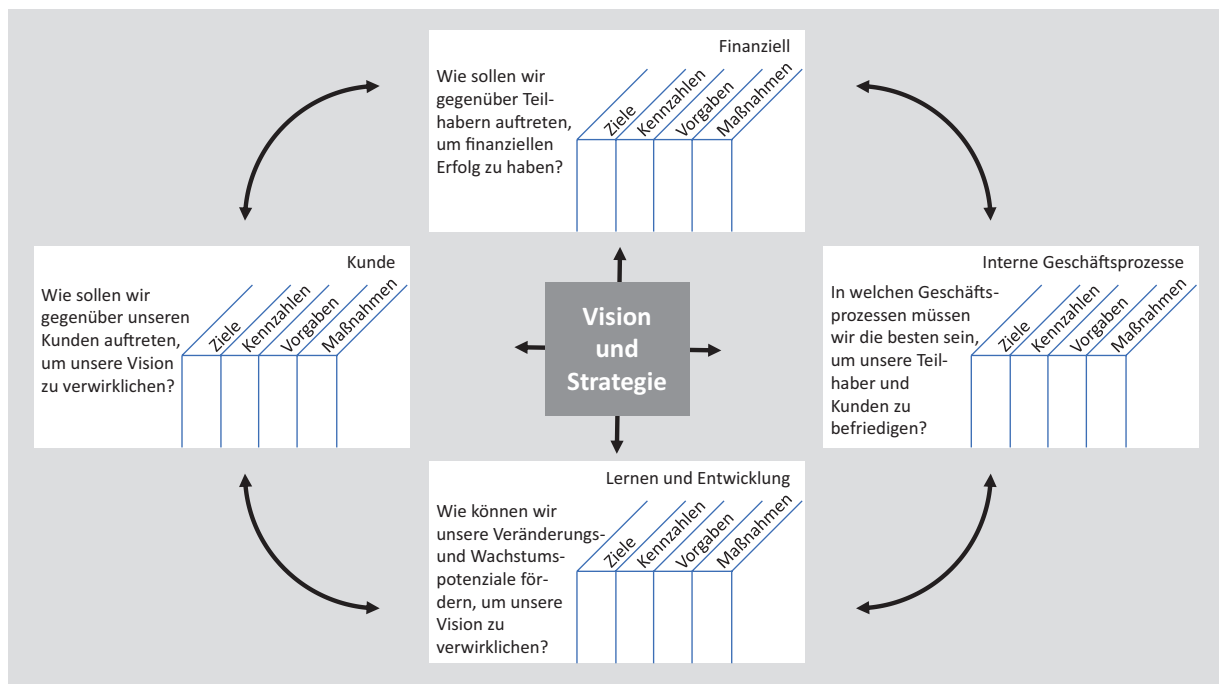

Abb. 2.4 Perspektiven der Balanced Scorecard [14] (Mit freundlicher Genehmigung von (C) Springer Fachmedien Wiesbaden GmbH 2010. All Rights Reserved) 
darum, auf die Interessen des Kunden zu fokussieren und Qualität an der Erfüllung seiner Anforderungen festzumachen.

Welche Anforderungen ein Unternehmen dabei an sich stellt, und durch welche Positionierung gegenüber dem Kunden es sich den größten und nachhaltigsten Geschäftserfolg verspricht, entscheidet die Führung eines Unternehmens selbst. Diese Positionierung ist nicht statisch. Erkenntnisse zu Kundenwünschen und zu den Vorgehensweisen zur Erfüllung dieser Wünsche erfordern eine permanente Anpassung des Unternehmens.

Um TQM zu etablieren bietet die European Foundation for Quality Management (EFQM) Organisationen Hilfestellung für den Aufbau und die kontinuierliche Weiterentwicklung eines umfassenden Managementsystems. Abb. 2.5 zeigt die Struktur des EFQM-Ansatzes. Diese Struktur dient zum einen als Werkzeug, um ein TQM aufzubauen und zum anderen dazu, durch ein umfassendes Bewertungssystem Verbesserungspotenziale zu ermitteln und den Geschäftserfolg zu steigern.

Als Befähiger gelten im EFQM-Modell die Methoden und Konzepte, die eingesetzt werden, um die Ergebnisse zu erreichen, die in der rechten Hälfte der Abbildung dargestellt sind. Die Prozentwerte in der Darstellung geben an, in wieweit die einzelnen Aspekte in die Gesamtbewertung eines Unternehmens einfließen.

Bei den Befähigern geht die EFQM davon aus, dass Prozesse den größten Einfluss auf die Ergebnisse auf der rechten Seite haben. Damit liefert das Modell gute Ansatzpunkte für die Identifikation von Kennzahlen und deren Zielwerte.

\section{Check, Act}

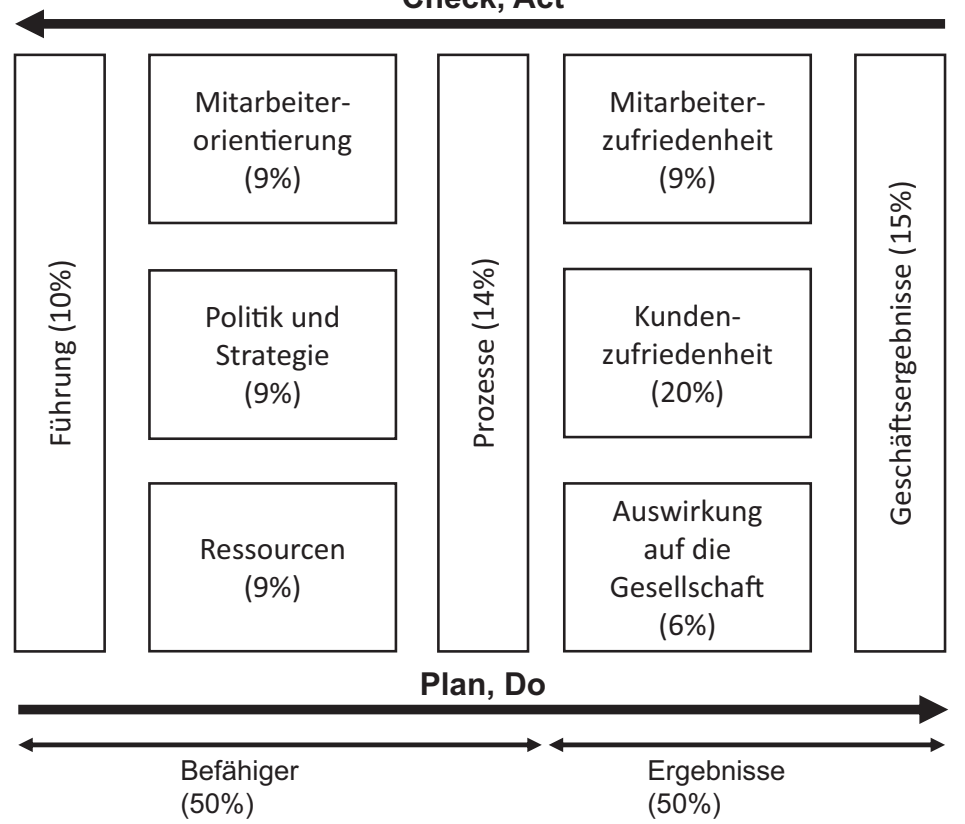

Abb. 2.5 EFQM-Struktur 
Neben der Möglichkeit ein Managementsystem aufzubauen bietet EFQM auch ein sehr ausgefeiltes Konzept, dessen Stand der Entwicklung zu bewerten. Durch einen umfangreichen Fragekatalog kann eine Rundumbewertung einer Organisation erfolgen. Die Bewertung kann durch Mitarbeiter der Organisation selbst oder durch externe Berater erfolgen. Die besten Organisationen in Europa erreichen bei einer solchen Bewertung rund 750 von maximal 1000 erzielbaren Punkten.

\subsubsection{EN ISO 9001}

Eine im Vergleich zu TQM abgeschwächte Form eines Qualitätsmanagements bildet der Standard EN ISO 9001. Er beschreibt Minimalanforderungen an ein Qualitätsmanagementsystem. Abb. 2.6 illustriert die Grundlagen des Standards.

Die Verantwortung der Leitung bedeutet, dass diese definiert, welche Kundenforderungen erfüllt werden und welche Qualitätspolitik dabei verfolgt wird. Die Umsetzung der Qualitätspolitik wird geplant und die entsprechenden Verantwortlichkeiten und Befugnisse in der Organisation dafür festgelegt. In der Verantwortung der Leitung liegt auch die Bewertung des QM-Systems in geplanten Abständen und insbesondere unter Berücksichtigung der Kundenrückmeldungen. Die Unternehmensführung muss außerdem die notwendigen Ressourcen wie Personal, Infrastruktur und eine adäquate Arbeitsumgebung bereitstellen.

Den Kern eines EN ISO 9001 konformen QM-Systems bilden die Prozesse zur Realisierung der Produkte und der zugehörigen kundenbezogenen Dienstleistungen. Aufgaben umfassen die Planung und Definition von geeigneten Prozessen für die Entwicklung und Herstellung von Produkten, die Beschaffung von Inputs usw. Die Hilfsmittel zur Überwachung der Produktherstellung und der Produktqualität müssen regelmäßig auf ihre Tauglichkeit überprüft werden. Die Ausführung der Prozesse muss durch Messungen

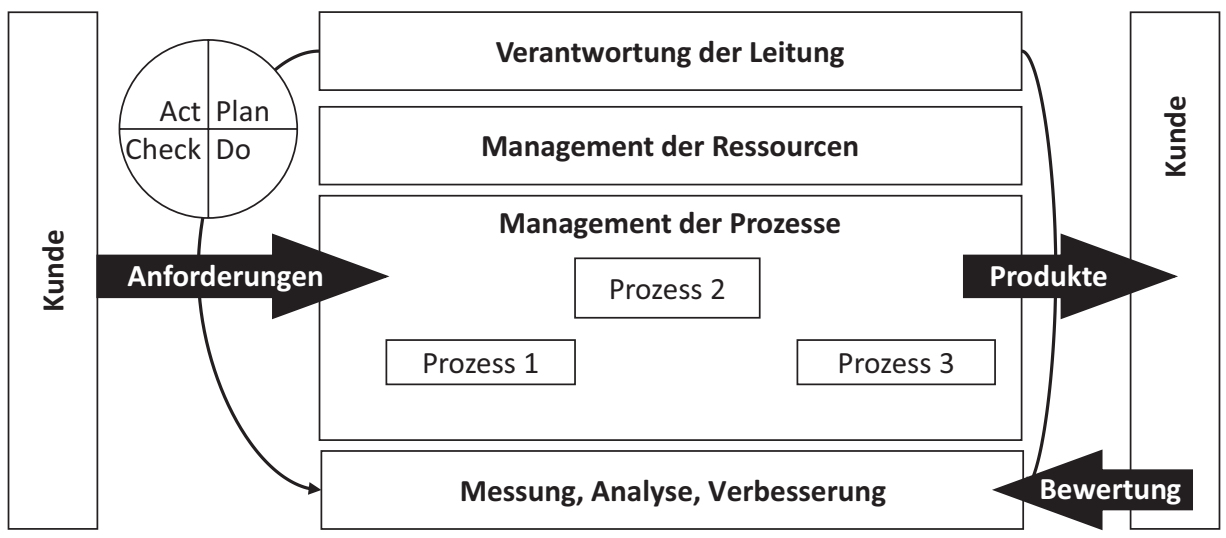

Abb. 2.6 EN ISO 9001 
und Analysen von Kennzahlen laufend überwacht werden, um bei Abweichungen entsprechende Verbesserungsmaßnahmen einleiten zu können.

EN ISO 9001 stellt also einen Rahmen für das Geschäftsprozessmanagement bereit. Die Ausführungen zeigen, dass es genau genommen keinen Unterschied zwischen Geschäftsprozess- und Qualitätsmanagement gibt. Ohne Geschäftsprozessmanagement gibt es kein Qualitätsmanagement und umgekehrt.

Die vergleichsweise niedrigeren Anforderungen von EN ISO 9001 äußern sich in der Tatsache, dass ein Unternehmen mit einem (nur) EN ISO 9001-konformen Qualitätsmanagementsystem etwa 300 Punkte bei einer EFQM Bewertung erreichen kann.

\subsubsection{Value Networks}

Das Konzept der Value Networks stammt von Verna Allee [15]. Unter einem Value Network versteht man Rollen und Personen, die untereinander sogenannte Tangibles und Intangibles austauschen. Tangible Wertflüsse sind materielle Wertflüsse zwischen Rollen und Personen und entsprechen dem Austausch von Waren, Dienstleistungen, Umsatzerlösen usw. Tangible Wertflüsse repräsentieren Transaktionen, die auf Verträgen basieren. Intangible Wertflüsse sind ein Zusatznutzen durch den Fluss von Wissen, sie sind nicht vertraglich fixiert oder kostenpflichtig. Intangible Wertflüsse sind z. B. strategische Informationen, Planungswissen sowie bestehende emotionale Komponenten wie gegenseitiges Vertrauen, gemeinsame Interessen, Wissensbedarf, Sicherheit usw.

Value Networks sollen Beteiligten und Organisationsentwicklern durch die Sichtbarmachung und gestalterische Handhabung wechselseitiger tangibler und intangibler Leistungsflüsse (Transaktionen) befähigen, soziale und fachliche Bezüge von Interaktion in organisationalen Systemen mit zu bestimmen bzw. aktiv zu gestalten. Abb. 2.7 zeigt

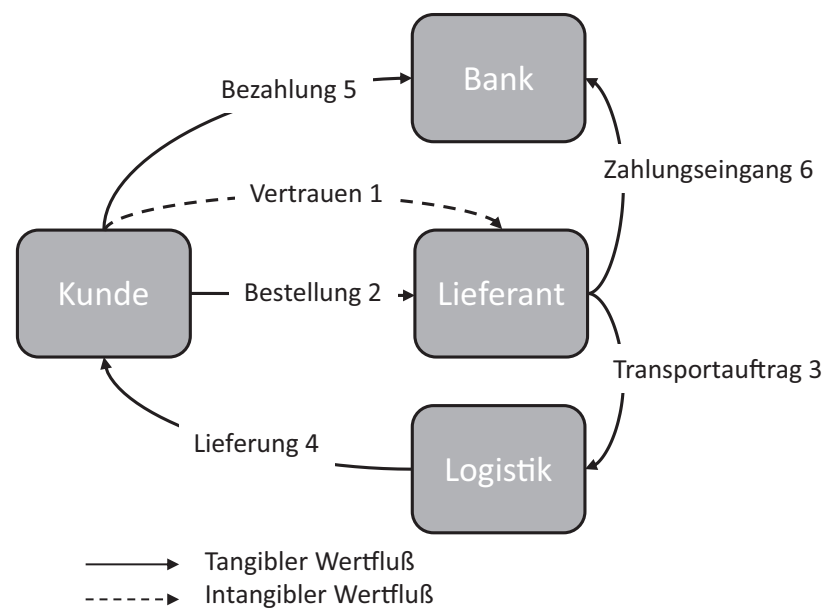

Abb. 2.7 Schema eines Value Network 
ein einfaches Value Network. Der Kunde gibt einen Wert Bestellung als einen tangiblen Wertfluss an den Lieferanten. Dieser tangible Wertfluss wird begleitet vom intangiblen Wertfluss Vertrauen. Der Kunde und die Logistikfirma sind mit dem tangiblen Wertfluss Lieferung verbunden usw. Die Nummern an den einzelnen Transaktionen drücken aus, in welcher Reigenfolge sie ausgeführt werden.

Organisationen erbringen Leistungen als Ergebnis ihrer Aktivitäten, die schließlich zur Wertschöpfung eines Unternehmens oder einer Institution beitragen. Um diese Leistungen zu erfassen und sichtbar zu machen, empfiehlt sich eine austauschorientierte Sicht auf Organisationen. Es ergibt sich dabei zumeist eine netzwerkartige Struktur, in der die Rollen innerhalb einer Organisation sowie deren Übertragungs- bzw. Kommunikationskanäle im Vordergrund stehen. Damit kann der Übergang zu einem kommunikationsorientierten Geschäftsprozessmanagement erfolgen.

\subsection{Modelle der Wirtschaftsinformatik}

Modelle der Wirtschaftsinformatik kombinieren Aspekte aus dem wirtschaftlichen und sozialen Bereich und der Informatik, um daraus Anforderungen für Informationssysteme abzuleiten. Die Modelle dienen überwiegend der Beschreibung von sozio-technischen Mensch-Maschine-Systemen. Die soziale Komponente deckt die Aspekte um die Mitarbeiter und Partner ab. Die technische Dimension betrifft die Sachverhalte der Informatik. Wichtig ist dabei, dass entsprechende Modelle die Wechselwirkung zwischen den beiden Domänen betrachten, insbesondere die Mensch-Maschine-Interaktion. Im Gegensatz zu rein technischen Systemen, die als deterministisch betrachtet werden, können sozio-technische Systeme aufgrund der Mitwirkung von sozialen Komponenten auch nicht-deterministisch, damit komplex, $\operatorname{sein}^{2}$.

Wir beschränken uns hier auf die Behandlung von Frameworks für Unternehmensarchitekturen und für IT-Management. Erstere entsprechen mit ihrer Verknüpfung der fachlichen und technischen Dimensionen von Organisationen genau dem Charakter der Wirtschaftsinformatik als Querschnittsdisziplin und sind von zentraler Bedeutung für das Prozessmanagement.

Frameworks für das IT-Management beziehen sich im Wesentlichen auf das IT-Service-Management und damit verbundene Aspekte wie Governance und Compliance. Die bekanntesten Vertreter sind die IT Infrastructure Library (ITIL ${ }^{\circledR}$ ) [17] und die Control Objectives for Information and related Technology (COBIT). Wegen seiner vergleichsweise weiten Verbreitung in der Praxis gehen wir näher auf ITIL $^{\circledR}$ ein und verweisen für weiterführende Informationen zu COBIT auf die einschlägige Website [18].

\footnotetext{
${ }^{2}$ Ausführliche Informationen zum Stand der Entwicklung und Forschung zum Thema Modellbildung in der Wirtschaftsinformatik finden sich beispielsweise in Franz Lehner; Modelle und Modellierung in der Wirtschaftsinformatik- Versuch einer Standortbestimmung [16].
} 


\subsubsection{Unternehmensarchitekturen}

Das Verständnis von Architektur im Kontext von Unternehmen deckt sich mit der ursprünglichen Bedeutung des Architekturbegriffs. Dieser bezeichnet in vielen Fachgebieten die grundlegende Organisation eines Systems mit seinen Komponenten und deren Beziehungen zueinander und zu ihrer Umgebung.

Konkret beschreibt und verknüpft eine Unternehmensarchitektur (Enterprise Architecture), wie bereits in Abschn. 1.4 angesprochen, die fachlichen und technischen Elemente des Unternehmens. Letztere umfassen insbesondere die IT-Landschaft. Sowohl die Gesamtarchitektur als auch ihre Teile werden mit Modellen beschrieben. Die Palette der dafür eingesetzten Modelltypen reicht vom Geschäftsmodell über Organigramme, Datenund Prozessmodelle auf der fachlichen Ebene bis hin zu Datenbankmodellen, Algorithmen und Programmen in der technischen Schicht.

Wie für Geschäftsmodelle gibt es auch für die Modellierung von Unternehmensarchitekturen zahlreiche Rahmenwerke, die den Verantwortlichen Orientierung geben und die Arbeit erleichtern sollen. Dirk Matthes [19] hat mehr als 40 Frameworks mit unterschiedlichem Detailierungsgrad, Schwerpunkt und Bekanntheitsgrad identifiziert.

Wir beschränken uns auf die Behandlung von vier einschlägigen Vertretern: Das Zachman-Framework und The Open Group Architecture Framework (TOGAF) mit seiner Ergänzung Architecture-Animate (ArchiMate) wurden in Umfragen wesentliche Rahmenwerke genannt [19]. Die Architektur Integrierter Informationssysteme (ARIS) ist im deutschsprachigen Raum in der Praxis weit verbreitet und hat signifikante Bedeutung im Rahmen des Prozessmanagements.

\subsubsection{Zachman-Framework}

Das von John A. Zachman 1992 in seiner erweiterten Form vorgestellte Framework stellt ähnlich dem Business Modell Canvas ein Strukturraster dar, das der Anwender mit den Fakten für sein Unternehmen anreichern muss. Es besteht aus einer Matrix mit verschiedenen Perspektiven in den Zeilen und Abstraktionen zu jeder Perspektive in den Spalten. Abb. 2.8 zeigt eine verdichtete Darstellung, ein detailliertes Bild findet sich auf der Website von Zachman International ${ }^{3}$.

Die Perspektiven in den Zeilen haben folgende Bedeutung:

- Planer: Zielsetzung des Unternehmens, externe Anforderungen und Einflüsse, Geschäftsmodell

- Besitzer: Anforderungen an Daten, Abläufe, Strukturen usw. zur Unternehmensführung

- Designer: Systementwurf und Systemstruktur zur Umsetzung der Anforderungen

\footnotetext{
${ }^{3}$ www.zachman.com.
} 


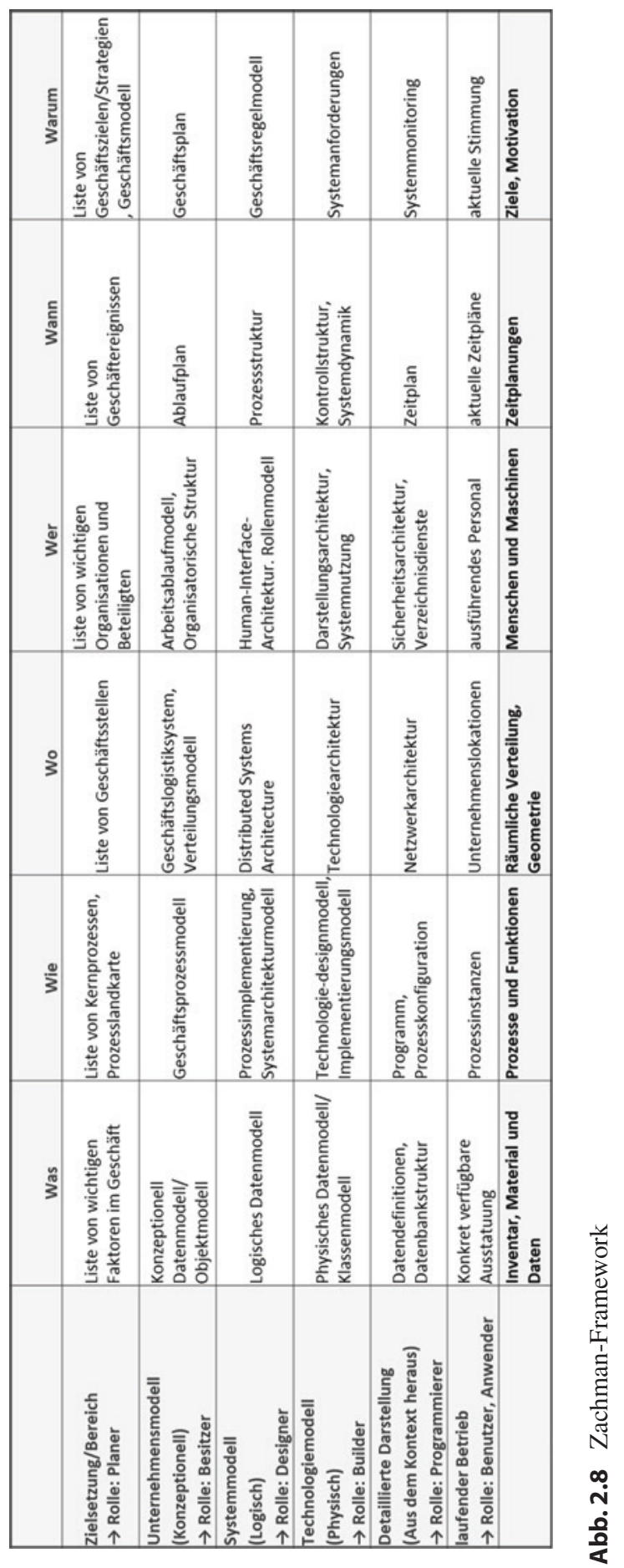


- Builder: Umsetzung des Systementwurfs

- Programmierer: Bereitstellen der technischen Infrastruktur

- Benutzer: Betriebsverantwortliche zur Sicherstellung der Funktionsfähigkeit

In den Spalten finden sich die Fragen, die das Unternehmen beantworten muss:

- Was (Inventar): Was für Objekte, Ausrüstungen, Daten, Informationen etc. werden benötigt?

- Wie (Funktionen und Prozesse): Wie arbeitet das Unternehmen, z. B. wie sehen die Geschäftsprozesse aus?

- Wo (Standorte, Netzwerk); Wo sind die Standorte des Unternehmens?

- Wer (Menschen): Wer sind die Menschen, die das Unternehmen am Laufen halten? Welche Geschäftseinheiten gibt es und wie sieht die Organisationsstruktur aus?

- Wann (Zeit): Wann werden Geschäftsprozesse instanziiert und ausgeführt? Was sind die Zeitpläne für das Geschäft?

- Warum (Motivation): Warum betreiben wir das Geschäft so wie wir es betreiben? Was sind die Treiber des Geschäfts? Hier fließen Aspekte des Geschäftsmodells ein.

Zachman sieht vor, dass für jede Zelle der Tabelle ein geeignetes Modell entwickelt wird. So gesehen handelt es sich bei seinem Framework um ein Modell für eine Menge von Modellen, die verschiedene Aspekte eines Unternehmens genauer betrachten.

Die Nutzer können bei den Reihen und Spalten durch andere Schwerpunktsetzung vom Original abweichen. Diese Flexibilität ist eine Stärke des Modellrahmens. Er enthält aber keine Vorgehensweise oder Methodik zur Definition einer konkreten Unternehmensarchitektur. Prozesse für deren Entwicklung oder Transformation müssen sich die Anwender anderweitig erschließen oder gänzlich selbst gestalten.

\subsubsection{The Open Group Architecture Framework (TOGAF)}

TOGAF ist das Rahmenwerk der Open Group zur Entwicklung von Unternehmensarchitekturen einschließlich der Geschäftsprozesse. Während das Zachman-Framework die zu betrachtenden Objekte betont und kaum Unterstützung für den Architekturentwicklungsprozess bietet, fokussiert TOGAF auf die Vorgehensweise zur Modellerstellung. Es stellt Methoden und Werkzeuge zur Verfügung, die bei Einführung, Erstellung, Gebrauch und Weiterentwicklung von Unternehmensarchitekturen helfen.

TOGAF unterscheidet bei der Beschreibung vier Teilarchitekturen:

- Business Architecture

Fachliche Aspekte der Unternehmensarchitektur

- Data Architecture

Logische und physische Strukturen der Daten und Ressourcen für deren Management. 
- Application Architecture

Verwendete Anwendungssysteme und deren Beziehungen untereinander sowie ihre Relevanz für das Geschäft des Unternehmens.

- Technology Architecture

Anforderungen an die Software und Hardware für das Management der Daten und die Ausführung der Anwendungssysteme. Dies schließt z. B. Laufzeitumgebungen, Netzwerke, Middleware und sonstige Betriebsinfrastrukturen ein.

Das TOGAF-Rahmenwerk setzt sich aus folgenden Komponenten zusammen:

- Architecture Development Method (ADM)

Methode und Vorgehensweise zur Entwicklung einer Unternehmensarchitektur

- ADM Guidelines and Techniques

Satz von Hilfsmitteln und Richtlinien, die die Anwendung des ADM unterstützen (z. B. Hilfsmittel für die iterative Verwendung des ADM)

- Architecture Content Framework

Strukturmodell um die Ergebnisse, die mit dem ADM erzeugt werden, einheitlich und konsistent zu definieren, strukturieren und darzustellen

- Enterprise Continuum

Modell zur Strukturierung eines möglichen Repository, das die jeweiligen Architekturen und die möglichen Lösungen wie Modelle, Muster, Architekturbeschreibungen etc. enthalten kann

- Reference Models

Grundmodelle, an denen sich spezifische Modelle für ein Unternehmen orientieren können. Dies sind das Technical Reference Model (TRM) und das Integrated Information Infrastructure Model

- Architecture Capability Framework

Verschiedenen Referenzmaterialien für die Entwicklung bestimmter ArchitekturmodelleDie Architecture Development Method (ADM) bildet als iteratives Prozessmodell den Kern von TOGAF (vgl. Abb. 2.9). Damit werden alle Architekturartefakte erzeugt. ADM kann auf mehreren Ebenen angewendet werden, sodass die Architekten unterschiedliche Detailierungsstufen der Unternehmensarchitektur definieren können. Mithilfe der anderen Komponenten werden die Ergebnisse dann beschrieben, strukturiert und abgelegt.

- Die Phasen der ADM sind:

Verschiedenen Referenzmaterialien für die Entwicklung bestimmter ArchitekturmodelleDie Architecture Development Method (ADM) bildet als iteratives Prozessmodell den Kern von TOGAF (vgl. Abb. 2.9). Damit werden alle Architekturartefakte erzeugt. ADM kann auf mehreren Ebenen angewendet werden, sodass die Architekten unterschiedliche Detailierungsstufen der Unternehmensarchitektur definieren können. Mithilfe der anderen Komponenten werden die Ergebnisse dann beschrieben, strukturiert und abgelegt. 


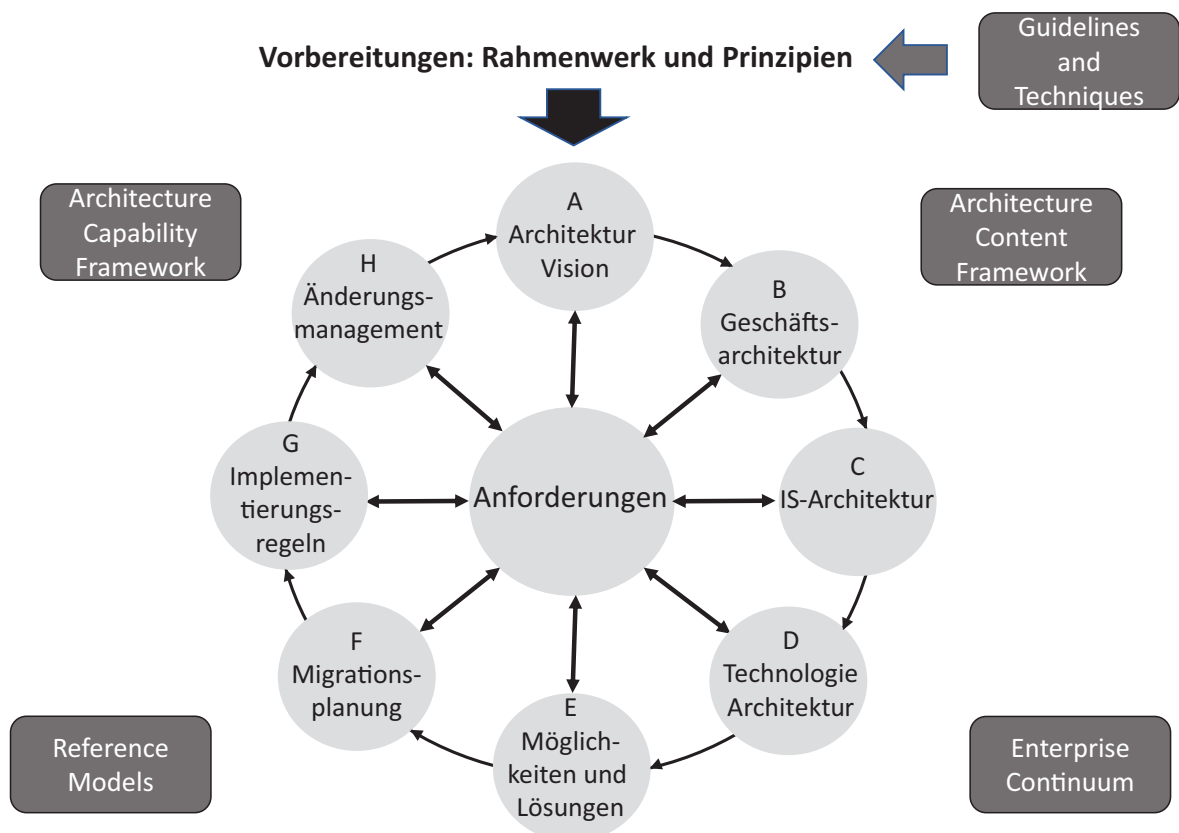

Abb. 2.9 Architecture Development Method von TOGAF

- Vorbereitungsphase (Preliminary Phase)

Hier werden das organisatorische Umfeld und die verwendeten Rahmenwerke, Methoden, Unterstützungswerkzeuge sowie wichtige Prinzipien festgelegt.

- Phase A Architekturvision (Architecture Vision)

Hier werden die Ziele und die Beteiligten bei der Aktualisierung der Unternehmensarchitektur festgelegt und eingebunden.

- Phase B Geschäftsarchitektur, Geschäftsmodell (Business Architecture)

Hier werden für die Geschäftsarchitektur der aktuelle und der gewünschte Zustand beschrieben. Die entscheidenden Unterschiede werden herausgearbeitet. Es werden die gewünschten Sichten festgelegt und die dazu geeigneten Werkzeuge ausgewählt.

- Phase C IS-Architektur (Information System Architecture)

Hier werden für die Anwendungs- und Informations-/Daten Architektur der aktuelle und der gewünschte Zustand beschrieben. Die entscheidenden Unterschiede werden herausgearbeitet. Dazu werden die konkreten Anwendungen und Datenmodelle verwendet.

- Phase D Technologiearchitektur (Technology Architecture)

Hier werden für die Technologiearchitektur der aktuelle und der gewünschte Zustand beschrieben. Die entscheidenden Unterschiede werden herausgearbeitet. Dazu werden die konkreten Hardwaresysteme beschrieben. 
- Phase E Möglichkeiten und Lösungen (Opportunities and Solutions)

Hier werden die Vorhaben festgelegt, welche die Transformation aus der Ist-Situation zum Sollzustand durchführen.

- Phase F Migrationsplanung (Migration Planning)

Hier wird die Überführung von einem Ist-Zustand in einen Sollzustand geplant.

- Phase G Implementierungsregeln (Implementation Governance)

Hier wird die Implementierung in den Sollzustand durchgeführt und überwacht.

- Phase H Änderungsmanagement (Architecture Change Management)

Hier werden Anforderungen und externe Einflüsse gesammelt, welche dann als Grundlage für den nächsten Durchlauf des ADM dienen.

- Anforderungen (Requirements Management)

Das Anforderungsmanagement treibt den ADM-Prozess kontinuierlich und steht deshalb im Zentrum.

\subsubsection{Architecture-Animate (ArchiMate)}

Architecture-Animate, kurz ArchiMate ${ }^{4}$, ist die Bezeichnung einer von der Open Group veröffentlichten offenen und unabhängigen Modellierungssprache für Unternehmensarchitekturen. Sie bietet Instrumente, die es Enterprise-Architekten ermöglichen, die Beziehungen zwischen Geschäftsbereichen und deren Entwicklung zu beschreiben, zu analysieren und zu visualisieren.

Die ArchiMate-Sprache ermöglicht die Beschreibung der Struktur und des Ablaufs von Geschäftsprozessen, von Organisationsstrukturen, Informationsflüssen, IT-Systemen und der technischen Infrastruktur. Die Beschreibungen helfen den Beteiligten, Veränderungen von Architekturelementen und deren Beziehungen zu konzipieren, die Folgen zu bewerten und zu kommunizieren. Abb. 2.10 zeigt das ArchiMate-Rahmenwerk.

Die ersten drei Spalten entsprechen den Grundkonzepten von ArchiMate:

- Passive Strukturelemente (Passive structure elements)

Passive Strukturelemente sind die Objekte, auf denen die Aktionen aus dem Verhalten (Verhaltenselemente) ausgeführt werden. Im Allgemeinen sind dies Informationsobjekte, aber auch physikalische Objekte können als passive Strukturelemente modelliert werden.

- Aktive Strukturelemente (Active structure elements)

Aktive Strukturelemente sind Elemente, die Handlungen ausführen können. Beispiele sind Menschen, Anwendungen, Rechnerknoten o. Ä. Die Handlungen können über Schnittstellen (Interfaces) angestoßen werden, die auch die Ergebnisse zur Verfügung stellen.

\footnotetext{
${ }^{4}$ http://www.opengroup.org/subjectareas/enterprise/archimate-overview.
} 


\begin{tabular}{|c|c|c|c|c|}
\hline & $\begin{array}{l}\text { Passive } \\
\text { structure }\end{array}$ & Behavior & Active Strucure & Motivation \\
\hline Strategy & ressourcen & & ressourcen & \multirow{6}{*}{$\begin{array}{l}\text { Stakeholders, } \\
\text { drivers, goals, } \\
\text { principles and } \\
\text { requirements }\end{array}$} \\
\hline Business & Business objects & $\begin{array}{l}\text { Business services, } \\
\text { functions and processes }\end{array}$ & Business actors and roles & \\
\hline Application & Data objects & $\begin{array}{l}\text { Application services, } \\
\text { functions and processes }\end{array}$ & $\begin{array}{l}\text { Application components and } \\
\text { interfaces }\end{array}$ & \\
\hline Technology & artifacts & $\begin{array}{l}\text { Technology services, } \\
\text { functions and processes }\end{array}$ & $\begin{array}{l}\text { Devices, system software, } \\
\text { communicatioon networks }\end{array}$ & \\
\hline Physical & material & & & \\
\hline $\begin{array}{l}\text { Implementation and } \\
\text { migration }\end{array}$ & deliverables & Work packages & platforms & \\
\hline
\end{tabular}

Abb. 2.10 ArchiMate Architectural Framework

- Verhaltenselemente (Behavior elements)

Verhaltenselemente repräsentieren die dynamischen Aspekte eines Unternehmens. Ein Service ist das von außen sichtbare Verhalten des Systems, das diesen Service erbringt. Die Services werden über die entsprechenden Schnittstellen verwendet. Schnittstellenereignisse stoßen die aktiven Strukturelemente an, welche dann die zugehörige Servicefunktion ausführen.

Diese drei Modellfragmente entsprechen den Grundelementen von natürlichen Sprachen: Subjekt, Prädikat bzw. Verb und Objekt. Sie werden auf insgesamt sechs Ebenen betrachtet:

- Strategieebene (Strategy Layer)

In der Motivation wird beschrieben, was ein Unternehmen erreichen möchte. In den Strategiekonzepten wird auf übergeordneter Ebene beschrieben wie ein Unternehmen seine Ziele erreichen möchte.

- Geschäftsebene (Business Layer)

Mit den Elementen des Business Layer können Produkte und Services beschrieben werden, die ein Unternehmen extern zur Verfügung stellt. Die Geschäftsebene zeigt, wie das Unternehmen diese Produkte und Services realisiert und soll bei der Analyse der Unternehmensstruktur helfen.

- Applikationsebene (Application Layer)

In der Anwendungsebene wird die Unterstützung der Geschäftsebene durch Anwendungen und Daten dargestellt.

- Technologieebene (Technology Layer)

Auf der Technologieebene wird die Infrastruktur beschrieben, welche benötigt wird, um Anwendungen zu realisieren. Dies sind im Wesentlichen die benötigten Hardwareund Softwarekomponenten. 
- Physikalische Ebene (Physical Layer)

In dieser Ebene wird auf das Zusammenwirken von IT und den physischen Komponenten wie Maschinen, Sensoren und Aktoren fokussiert.

- Implementierung und Migration (Implementation and Migration Layer)

Das Implementierungs- und Migrationskonzept beschreibt, wie eine definierte Architektur realisiert werden soll. Insbesondere werden darin die Arbeitspakete für die Umsetzung beschrieben.

Die Zellen der Tabelle enthalten also die Kernelemente für die aktiven und passiven Strukturen sowie für das Verhalten auf der jeweiligen Ebene.

Die Spalte Motivation beschreibt die Gründe für den Entwurf oder die Änderung eines Unternehmensmodells. Diese beeinflussen die Modellbildung und geben ihr die entsprechende Richtung.

ArchiMate wird als Ergänzung und Konkretisierung von TOGAF gesehen. TOGAF beschreibt den Prozess für die Definition und Beschreibung einer Unternehmensarchitektur (Unternehmensmodell), allerdings enthält es keine Beschreibungssprachen für die jeweiligen Teilmodelle. ArchiMate soll diese Lücke schließen. Es bietet neben dem gezeigten Rahmenwerk für die zu entwickelnde Architektur auch Sprachen, um die Aspekte in den einzelnen Ebenen auszudrücken. Abb. 2.11 zeigt wie die einzelnen Schritte in der Architecture Development Method von TOGAF mit den Schichten von ArchiMate zusammenhängen.

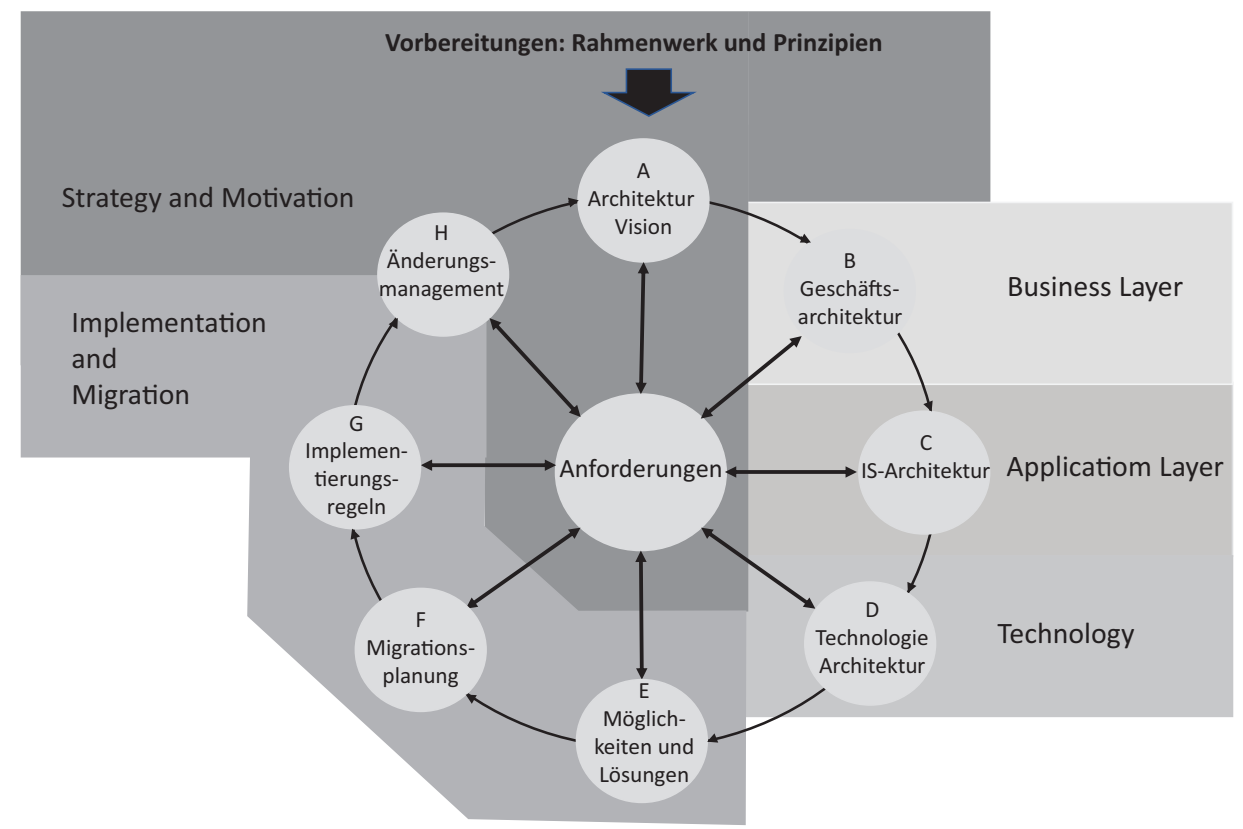

Abb. 2.11 Zusammenhang von ArchiMate-Ebenen und TOGAF ADM 


\subsubsection{Architektur Integrierter Informationssysteme (ARIS)}

Die Architektur integrierter Informationssysteme (ARIS) ist ein Rahmenwerk zur Definition von Unternehmensmodellen. Es umfasst die Daten-, Funktions-, Organisations-, Steuerungs- und Leistungssicht. Für jede Sicht sieht ARIS eine Reihe von Modelltypen für die Dokumentation vor.

- Datensicht:

Die Datensicht umfasst die betriebswirtschaftlich relevanten Geschäfts- oder Informationsobjekte und deren Beziehungen untereinander, das heißt alle Daten, die im Zusammenhang mit den Aktivitäten eines Unternehmens stehen. Informationsobjekte umfassen sowohl Zustände wie Artikel- oder Kundenstatus als auch Ereignisse wie „Kundenauftrag ist eingetroffen“ oder „Fertigungsauftrag wurde ausgelöst“. Einschlägiger Modelltyp ist das Entity Relationship Model (ERM).

- Funktionssicht:

Die Funktionssicht beschreibt die betriebswirtschaftlich relevanten Tätigkeiten (Funktionen, Aktivitäten) sowie ihre hierarchischen Beziehungen. Untergeordnete Funktionen sind Teilfunktionen der übergeordneten Funktion. Die Funktionen führen Operationen auf den in der Datensicht beschriebenen Objekten aus. Funktionen werden in der Praxis mit Funktionsbäumen modelliert.

- Organisationssicht:

In der Organisationssicht wird die Organisationsstruktur, das heißt die personellen Ressourcen, eines Unternehmens und deren typischerweise hierarchischen Beziehungen in einer Aufbauorganisation modelliert. Das Organigramm ist hierfür als Modelltyp üblich.

- Steuerungssicht:

Die Steuerungssicht stellt den zeitlich-sachlogischen Zusammenhang zwischen den einzelnen betrieblichen Tätigkeiten her. Sie führt die Daten-, Funktions- und Organisationssicht zusammen und nimmt damit eine zentrale, integrierende Rolle ein. Die Steuerungsschicht wird deshalb auch Prozesssicht genannt. Wesentlicher Modelltyp ist die Ereignisgesteuerte Prozesskette (EPK).

- Leistungssicht:

In der Leistungssicht werden die Eingaben und Ergebnisse des beschriebenen Geschäftsprozesses in der Regel mithilfe von Produktbäumen beschrieben.

Die Sichten, dafür typische Modelltypen und ihr Zusammenhang sind in Abb. 2.12 dargestellt. Das Bild macht auch deutlich, dass ARIS mit der integrierenden Steuerungssicht die Geschäftsprozesse, insbesondere mit der Abfolge der Aktivitäten, in den Mittelpunkt der Betrachtung stellt.

Orthogonal zu der durch die Sichten vorgenommenen Strukturierung differenziert ARIS in Anlehnung an das Software Engineering die Abstraktionsebenen Fachkonzept, DV-Konzept und Implementierung. Dies zeigt die Nähe der mit ARIS entwickelten Modelle zur Informationstechnik. Für die Lösung einer betrieblichen Problemstellung 


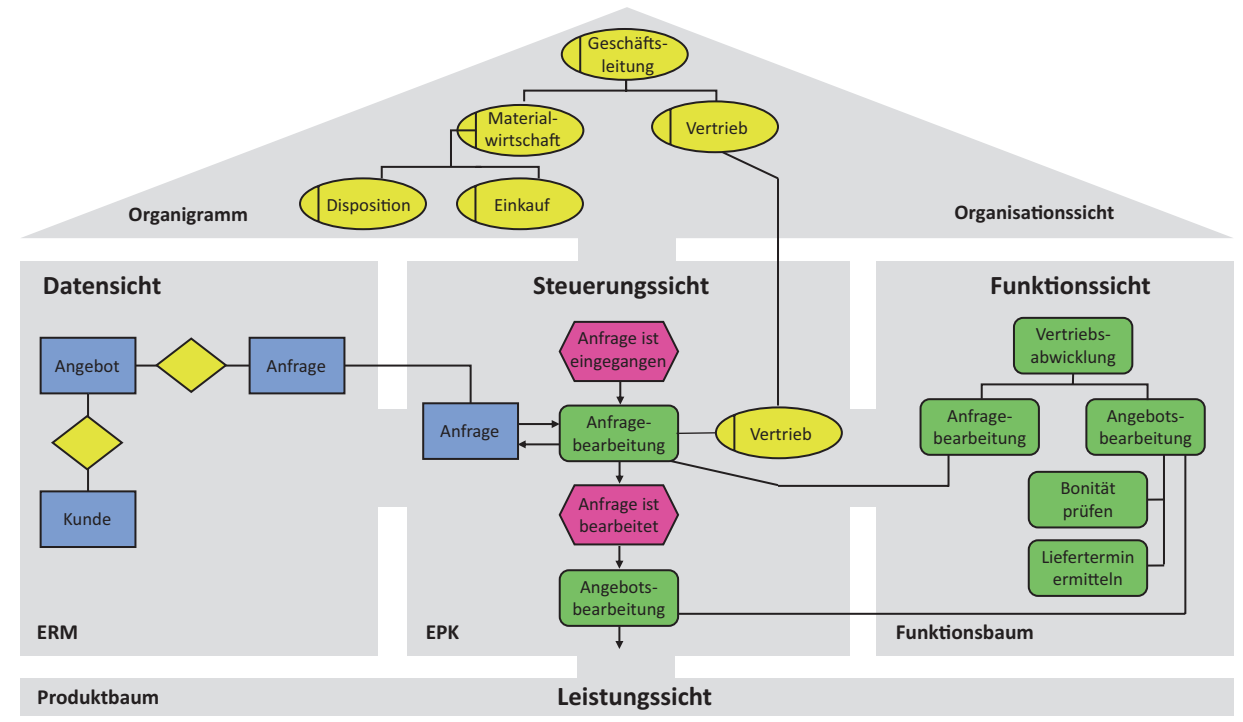

Abb. 2.12 Sichten und einschlägige Modelltypen in ARIS

wird ein fachliches Modell erstellt und in ein entsprechendes DV-Konzept (DV-Modell) überführt. Dieses dient schließlich als Basis für die konkrete technische Umsetzung.

- Fachkonzept:

Im Fachkonzept werden die Sachverhalte der betrieblichen Problemstellung beschrieben. Auf dieser Ebene werden Datenmodelle, Funktionsmodelle, Organigramme, Wertschöpfungsketten bzw. Ereignisgesteuerte Prozessketten (EPKs) und Produktmodelle eingesetzt.

- DV-Konzept:

Im DV-Konzept wird spezifiziert, wie das Fachkonzept DV-technisch umzusetzen ist. Auf dieser Ebene werden Datenbankmodelle (Datensicht), Struktogramme (Funktionssicht), Netztopologien (Organisationssicht) und Trigger-Mechanismen (Steuerungssicht) betrachtet. Der Zweck des DV-Konzepts ist eine Anpassung des Fachkonzepts an die Anforderungen der Informationstechnik.

- Implementierung:

Auf dieser Ebene wird das DV-Konzept in ein ausführbares Softwaresystem umgesetzt. Auf dieser Abstraktionsstufe werden Datenbeschreibungssprachen (Datensicht), Programme (Funktionssicht), Netzwerkprotokolle (Organisationssicht) und die Programmsteuerung (Steuerungssicht) betrachtet. 


\subsubsection{Framework für IT-Service-Management: ITIL}

Die IT Infrastructure Library $\left(\right.$ ITIL $\left.^{\circledR}\right)$ ist eine Sammlung vordefinierter Prozesse, Funktionen und Rollen, wie sie typischerweise in jeder IT-Infrastruktur mittlerer und großer Unternehmen vorkommen. Die praktische Zuweisung der Tätigkeiten erfolgt anhand von Rollen und Funktionen. Es handelt sich dabei um Good-Practice-Vorschläge, die an die Bedürfnisse des Unternehmens angepasst werden müssen. Inzwischen wurde die Sammlung ergänzt durch ISO 20.000:2005, ein ITIL $^{\circledR}$-basiertes Zertifizierungsmodell für Organisationen.

ITIL $^{\circledR}$ umfasst fünf Kernbände mit derzeit insgesamt 37 Kernprozessen. Abb. 2.13 zeigt die Struktur von ITIL $^{\circledR}$. Die fünf Kernbände orientieren sich am Service Live Cycle. Ausgehend von der Servicestrategie mit den Prozessen Strategiefindung, Finanzmanagement, Serviceportfoliosteuerung und Nachfragesteuerung wird über die Prozessgruppen der Serviceentwicklung und der Serviceinbetriebnahme schließlich die Dienstleistung mit den Prozessen der Gruppe Serviceerbringung erbracht. Die Prozeduren unterliegen der permanenten Verbesserung.

Weitere Bücher wie „Software Asset Management“, „Small-Scale Implementation“ oder „Building an ITIL ${ }^{\circledR}$-based Service Management Department“ ergänzen die Kernpublikationen. Damit bietet ITIL ${ }^{\circledR}$ umfassende Unterstützung bei der Entwicklung eines Prozesssystems für den IT-Bereich einer Organisation.
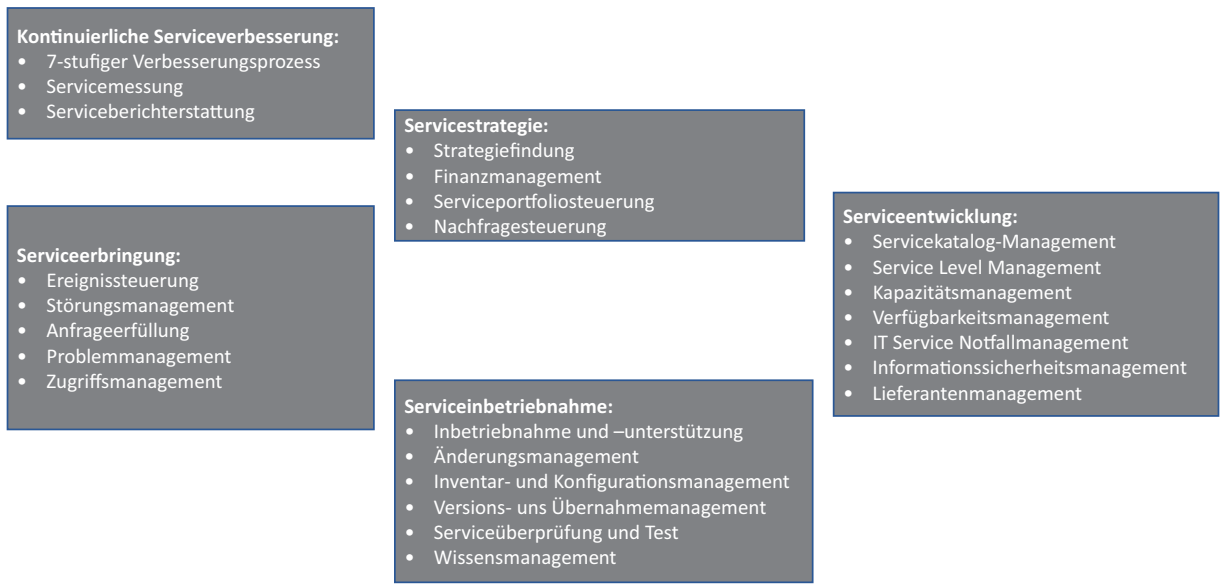

Abb. 2.13 ITIL ${ }^{\circledR}$-Prozessgruppen 


\subsection{Modelle der Informatik}

Modelle der Informatik beziehen sich auf Datenstrukturmodelle und Abläufe in Computersystemen sowie wesentliche Aspekte wie Sicherheit, Robustheit etc.

Diese Modelle dienen zum einen zur Abbildung eines betrachteten Realitätsausschnitts, um eine Aufgabe mithilfe der Informationsverarbeitung zu lösen. Sie beziehen sich auf ein abgegrenztes Problemfeld oder bestimmte Einsatzbereiche von Computersystemen. Hierunter fallen Modelle, die Daten und die darauf ablaufenden Operationen im Fokus haben, sowie Modelle, die mehr die Gesamtstruktur von komplexen Programmen, also deren Architektur, betrachten. Diese beiden Modellkategorien werden auch Modelle zum Programmieren im Kleinen bzw. Modelle zum Programmieren im Großen genannt.

Neben diesen zentralen Modellkategorien der Informatik gibt es weitere Modelle, die flankierende Aspekte betrachten, wie Zugriffsmodelle, Sicherheitsmodelle usw. Diese sind nicht Gegenstand der weiteren Ausführungen. Vielmehr erläutern wir in den folgenden Abschnitten einige für das Geschäftsprozessmanagement wesentliche Modellkonzepte.

\subsubsection{Information}

Information ist der zentralere Aspekt der Datenverarbeitung, die deshalb auch Informationstechnologie oder IT genannt wird. Information und Informationssysteme sind Modelle, die ein Objekt der realen Welt gemäß den Vorstellungen eines oder mehrerer Subjekte repräsentieren. Die Vorstellungen des Subjekts orientieren sich dabei am beabsichtigten Verwendungszweck. Beispielsweise werden Gegenstände in einem Lager durch ihre Eigenschaften beschrieben wie Teilenummer, Maße, Gewicht usw. Verschiedene Anwender verwenden unterschiedliche Ausschnitte aus dem Modell: Der Einkauf blickt auf Informationen wie Einkaufspreis und Bestellgrenze, während den Lagerarbeiter eher die Maße und der Lagerplatz interessieren.

Die Modellierung der Objektrealität kann deshalb als Interpretation durch die verwendenden Handelnden (Subjekte) wie Einkauf oder Lagerist verstanden werden. Information ist dann das Ergebnis und der Grund einer Interpretation; Sie kann aber auch selbst wiederum Objekt und damit Interpretations- und Modellierungsgegenstand sein. Dieses Verhältnis zwischen Subjekt, Information (Modell) und Original ist in Abb. 2.14 dargestellt:

Information erhält ihren Wert durch die Interpretation des Gesamtgeschehens durch die betrachtenden Subjekte. Diese Betrachtung läuft teils bewusst, größtenteils aber unbewusst ab. Die Informationsmenge wird dabei reduziert und dem jeweiligen Erkenntnisinteresse entsprechend gefiltert oder mit anderen Informationen verknüpft.

Daten unterscheiden sich von Informationen. Ein Datum (Einzahl von Daten) stellt zunächst nur eine Folge von Zeichen dar, deren Bedeutung nicht eindeutig ist. Die Zeichen können z. B. Zahlen, Buchstaben oder Symbole sein. In der Marketingabteilung 
eines Online-Shops findet sich beispielsweise die Zahlenfolge 0815. Diese Abfolge von Zeichen stellt zwar ein Datum dar, die Bedeutung ist allerdings nicht bekannt. Die Zeichenfolge selbst hat keine Bedeutung mit Ausnahme ihrer einzelnen Elemente.

Aus diesem Datum können aber Informationen entstehen, wenn bekannt ist, in welchem Kontext es steht. Durch eine Kombination mit anderen Daten wird eine Beziehung hergestellt, die interpretiert werden kann und Information entstehen lässt. Steht das Datum 0815 im Kontext „Kunde Max Muster, Artikel 0815“ kann die Marketingabteilung interpretieren, dass der Kunde Max Mustermann einen Artikel mit der Nummer 0815 bestellt hat.

Die Ergänzungen von Daten mit anderen Daten hängen vom Erkenntnisinteresse bzw. Nutzungsabsichten eines Subjekts ab. Ein Subjekt möchte mit den jeweils hergestellten Informationen meist einen Adressaten beeinflussen, z. B. zu einer Handlung bewegen. Information ist somit zu verstehen als „Aussagen, die den Erkenntnis- bzw. Wissensstand eines Subjektes (Informationssubjekt/-benutzer) über ein Objekt (Informationsgegenstand) in einer gegebenen Situation und Umwelt (Informationsumwelt) zur Erfüllung einer Aufgabe (Informationszweck) verbessern“ [20] (vgl. Abb. 2.14). Die Modellbildung ist demnach ein Teil des Informationsmanagements.

Informationen sind wichtig für Politiker, Führungskräfte in der Wirtschaft aber auch für jeden Bürger dieser Welt. Sie geben einen bestimmten Sachverhalt, der zu einem bestimmten Zeitpunkt galt, wieder und erlauben in der Regel eine Fortschreibung in die Zukunft. Sie dienen dazu politische, wirtschaftliche oder persönliche Entscheidungen zu treffen. Bei der Nutzung von Informationen stellt sich immer die Frage, wer sie erzeugt hat und welche Absichten derjenige damit verfolgt. Hier spielt folglich die Subjektivität von Modellen eine wesentliche Rolle.

\subsubsection{Entity-Relationship-Modell}

Um aus Daten Informationen zu generieren, müssen diese wie gezeigt mit anderen Daten kombiniert und die entsprechenden Beziehungen beschrieben werden. Dadurch entsteht ein Datenmodell. Die bekannteste Methode für die Beschreibung von Datenmodellen ist

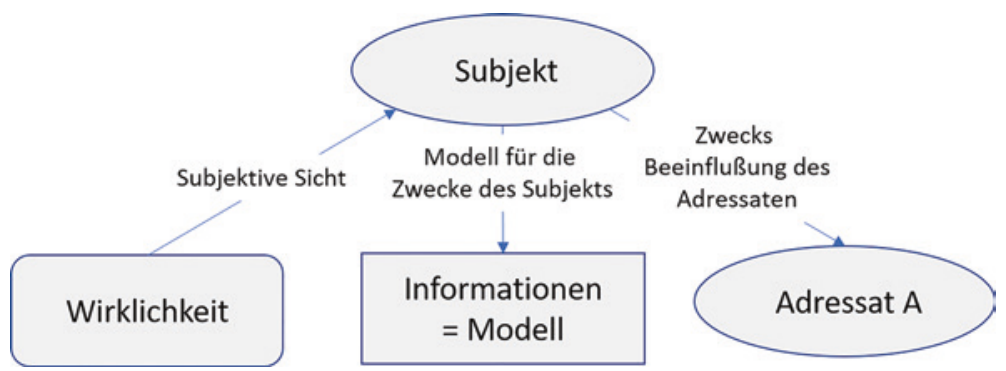

Abb. 2.14 Information ist „Modell-wovon-wozu-für wen“ [21] 


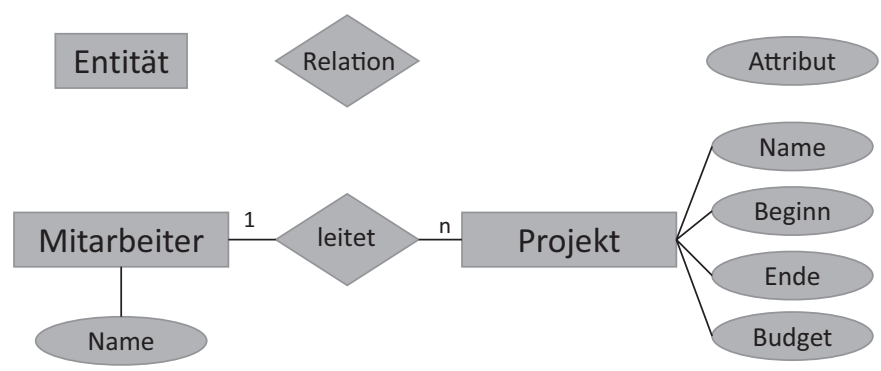

Abb. 2.15 Beispiel für ein Entity-Relationship-Diagramm

das Entity Relationship Model (ERM). Ein Entity-Relationship-Modell setzt sich aus drei Hauptelementen zusammen:

- Entitäten

Entitäten sind die Objektklassen, die in dem interessierenden Ausschnitt der realen Welt betrachtet werden.

- Beziehungen oder Relationen

Relationen beschreiben die Beziehungen zwischen Entitäten.

- Attribute

Attribute sind Eigenschaften innerhalb des Kontexts einer Entität.

Abb. 2.15 zeigt ein Beispiel für ein ERM. Aus ihm geht auch hervor, mit welchen Symbolen die Hauptelemente in der Regel ausgedrückt werden. Man findet jedoch auch ERM-Darstellungen mit Notationselementen der Unified Modeling Language (UML).

Das Diagramm beschreibt den folgenden Sachverhalt:

- Ein Mitarbeiter hat einen Namen. Ein Projekt hat einen Namen, einen Beginn, ein Ende und ein Budget. Die sogenannte Kardinalität drückt aus, dass ein Mitarbeiter mehrere Projekte leiten kann, aber ein Projekt nur von genau einem Mitarbeiter geleitet werden kann.

Im Modellierungskonzept der Klassifikation werden Objekte zu Objekttypen (entity sets), und Beziehungen zu Beziehungstypen (relationship sets) zusammengefasst. Diese Typen unterscheiden sich nach:

- Entitätstyp: Typisierung gleichartiger Entitäten (z. B. Mitarbeiter, Projekt)

- Beziehungstyp: Typisierung gleichartiger Beziehungen (z. B. Mitarbeiter leitet Projekt)

- Attributtyp: Typisierung gleichartiger Eigenschaften (z. B. Name für den Entitätstyp Mitarbeiter). Attribute oder Attributkombinationen, deren Wert(e) eine Entität eindeutig identifizieren, heißen identifizierende(s) Attribut(e) (z. B. identifiziert das Attribut Projektname den Entitätstyp Projekt) 
Terminierungselement

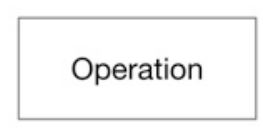

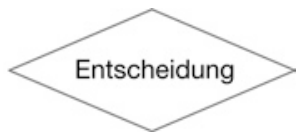

Abb. 2.16 Häufige Symbole in Flussdiagrammen

Zur Beschreibung besonderer Sachverhalte wie z. B. der angesprochenen Kardinalität umfassen ERMs noch weitere Konstrukte ${ }^{5}$.

\subsubsection{Fluss- oder Ablaufdiagramme}

Fluss- oder Ablaufdiagramme veranschaulichen eine Ausführungsreihenfolge von Tätigkeiten bzw. Aktionen und werden in zahlreichen Anwendungsbereichen verwendet. Sie können beschreiben, in welcher Reihenfolge Handlungen von Menschen oder anderen Akteuren ausgeführt werden sollen. Algorithmen oder Computerprogramme werden oft in Form von Flussdiagrammen (z. B. Programmablaufplänen) dokumentiert. Wegen der verbreiteten Anwendung von Flussdiagrammen haben sich zahlreiche Varianten herausgebildet, die spezielle Sachverhalte des jeweiligen Anwendungsbereichs berücksichtigen. Für die Datenverarbeitung wurde die Symbolik für Flussdiagramme in den Standards DIN 66.001:1983-12 bzw. ISO 5807:1985 festgelegt. Abb. 2.16 und 2.17 enthalten eine Auswahl häufig verwendeter Symbole und ein Beispiel für ein Flussdiagramm.

Bei der Erläuterung von ARIS in Abschn. 2.5.1.4 haben wir Ereignisgesteuerte Prozessketten (EPK) als Modelltyp für die Steuerungssicht angesprochen. Bei diesen EPKs handelt es sich um Flussdiagramme aus Sequenzen von Ereignisknoten, Funktionsknoten (Operationen) und Konnektoren. Pfeile als Kanten repräsentieren Kontrollflüsse zwischen den Symbolen. Funktionen und Ereignisse (mit Ausnahme von Start- und Endereignis) besitzen genau je eine eingehende und eine ausgehende Kante. Sollen Funktionen mehrere Ereignisse erzeugen oder mehrere Ereignisse eine Funktion auslösen, müssen Konnektoren wie ein exklusives Oder (XOR) zwischengeschaltet werden. Der Modellierer kann auch ausdrücken, wer eine Funktion mit welcher IT-Unterstützung ausführt und welche Daten dabei manipuliert werden. Dazu ordnet er den Funktionsknoten Symbole für Organisationseinheiten (z. B. Abteilungen, Stellen, Rollen), Informationsobjekte (Daten) oder Anwendungssysteme zu. Diese Elemente müssen in den entsprechenden Modelltypen spezifiziert sein. Man spricht dann von erweiterten Ereignisgesteuerten Prozessketten (eEPK).

${ }^{5}$ Details dazu finden sich in einschlägiger Literatur, z. B. bei [22]. 
Abb. 2.17 Flussdiagramm für Kontotransaktion

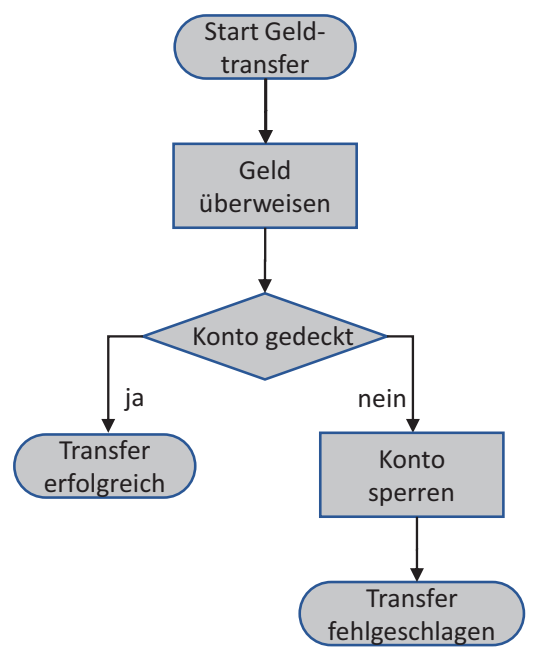

Den Zusammenhang, den eine eEPK als Instrument der Steuerungs- oder Prozesssicht mit den Elementen der anderen Sichten und Modelltypen herstellt, haben wir bereits in Abschn. 2.5.1.4 kurz angedeutet. Konkret äußert er sich folgendermaßen:

- Steuerungssicht

- Ein Ereignis ist ein Zustand, der vor oder nach einer Funktion auftritt. Das Symbol für ein Ereignis ist sechseckig.

- Eine Funktion (Prozess) ist eine Aktion oder Aufgabe, die auf ein Ereignis folgt. Funktionen werden durch Rechtecke mit abgerundeten Ecken symbolisiert.

- Konnektoren dienen zum Aufspalten (Split) oder Vereinigen (Join) des Kontrollflusses. Es stehen die drei Konnektoren UND, ODER und XOR zur Verfügung, die jeweils in einem kleinen Kreis mit dem entsprechenden Symbol dargestellt werden. Die Entscheidung, welcher Pfad nach einem Konnektor verfolgt wird, trifft die dem Konnektor vorangehende Funktion.

- Funktionssicht

- Die Funktionsknoten in der Steuerungssicht werden mit Knoten aus dem Funktionsbaum der Funktionssicht verknüpft - damit wird jeweils die auszuführende Aktivität spezifiziert.

- Datensicht

- Informationsobjekte sind Entitäten aus dem Datenmodell, die an Träger wie Dokumente oder sonstige Datenspeicher gebunden sind. Sie stellen und Inputs oder Outputs der Funktion dar, mit der sie durch eine gerichtete Kante verbunden werden. Das Symbol für ein Informationsobjekt ist ein Rechteck, der Charakter als Input oder Output bestimmt die Pfeilrichtung der Verbindungskante. 


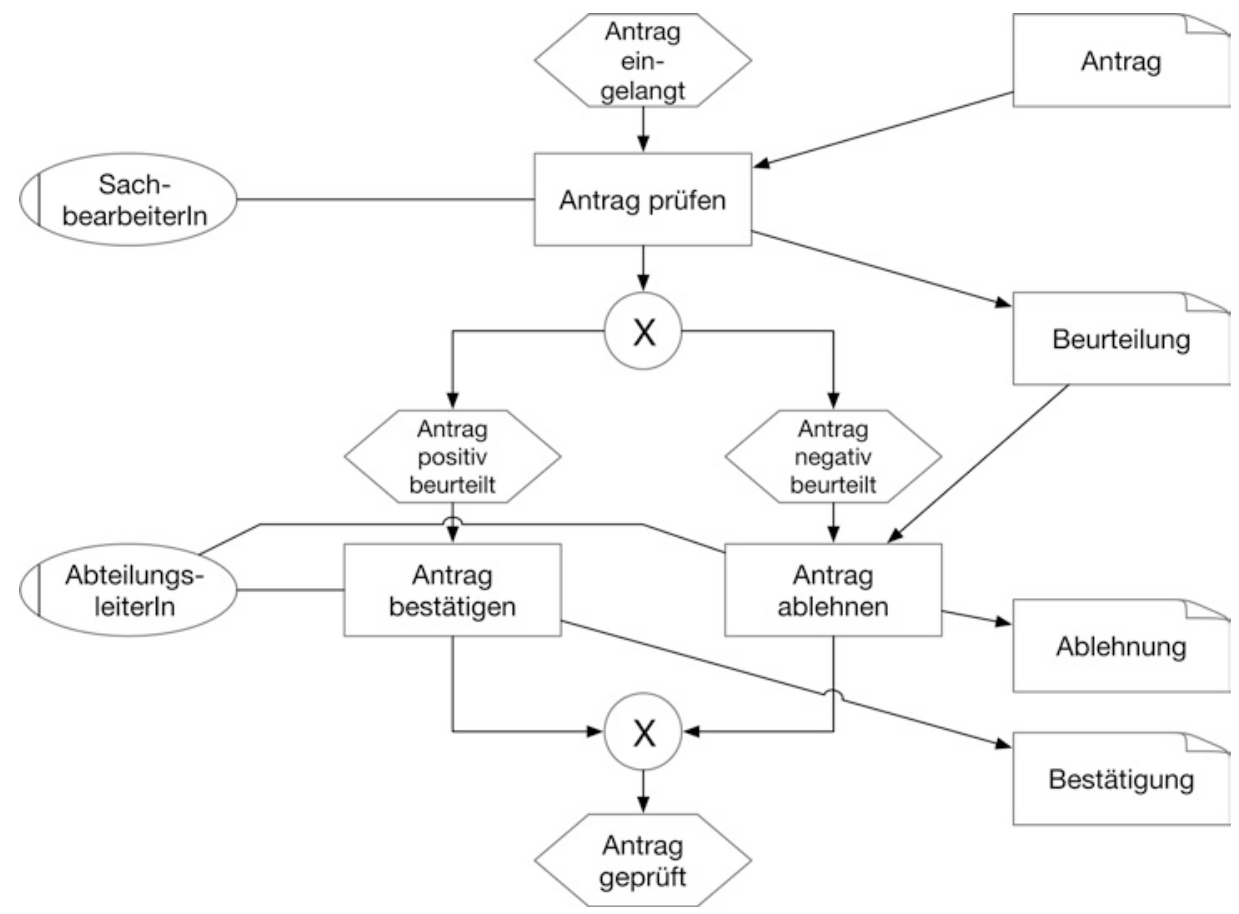

Abb. 2.18 Erweiterte Ereignisgesteuerte Prozesskette für Kontotransaktion

- Organisationssicht

- Organisationseinheiten zeigen an, welche Elemente aus dem für die Organisationssicht modellierten Organigramm die Aktivitäten (Funktionen) im Prozess ausführen. Organisationseinheiten werden durch ungerichtete Kanten mit Funktionen verbunden.

In Abb. 2.18 ist das Flussdiagramm aus Abb. 2.17 in der Notation einer erweiterten Ereignisgesteuerten Prozesskette zu sehen.

\subsubsection{Petrinetze}

Einer der ersten und auch am weitest verbreiteten Theorieansätze zur Beschreibung von Parallelität sind Petrinetze.

Petrinetze dienen der logischen Modellierung von Verhalten. Sie betrachten das Verhalten von Systemen, in der Regel von Informationssystemen unter folgenden Aspekten:

- Auszuführende Aktivitäten

- Vor- und Nachbedingungen einer Aktivität 
- Zustände aller Bedingungen (mögliche Ausprägung für jede einzelne Vor- bzw. Nachbedingung: Zustand einer Bedingung ist die Verteilung sogenannter Marken als Zustandsanzeige auf den Vor- bzw. Nachbereich)

- Anfangszustand (Anfangsmarkierung)

- Abläufe (mögliche Folgen von Aktivitäten)

Ein Petrinetz ist ein Gebilde, das formal und mathematisch präzise beschrieben wird als gerichteter Graph mit Knoten, die aus zwei disjunkten Teilmengen bestehen und mit Markierungen versehen sind. In der Schreibweise als Quadrupel gilt: Ein Petri Netz ist ein Quadrupel: $\mathrm{PN}=(\mathrm{S}, \mathrm{T}, \mathrm{K}, \mathrm{M}) \mathrm{mit}$

- $s$ \& S: Stellen (oder auch Plätze), zur Beschreibung von Zuständen und/oder Bedingungen, Puffer, Speicher oder Lager, im Graph kreisförmig. Sie dienen der Ablage von Information bzw. der Markierungen.

- $\quad \mathrm{t} \varepsilon$ T: Transitionen, beschreiben Zustandsübergänge, Ereignisse, Aktionen oder Tätigkeiten und sind im Graph strich-, balken- oder quaderförmig. Ihr Zweck ist die Verarbeitung von Information.

- $\mathrm{k} \varepsilon \mathrm{K}$ : Kanten sind ggf. gewichtete (d. h. mit Zahlen versehene) Verbindungen zwischen Stellen und Transition, im Graph als Pfeile dargestellt. Sie zeigen den Verlauf der Transitionen an.

- $\mathrm{m} \varepsilon$ M: Marken (Token), die den aktuellen Zustand des Petrinetzes wiedergeben. Jedes Netz hat einen Initialzustand, d. h. es gibt eine Anfangsmarkierung.

Gemäß obiger Definition gehen Kanten jeweils nur von Stelle zu Transition bzw. Transition zu Stelle. Eingabestellen einer Transition t sind Stellen, von denen Kanten zur Transition t laufen. Ausgabestellen einer Transition t sind Stellen, zu denen Kanten der Transition $\mathrm{t}$ führen.

Eine Transition kann schalten, wenn in jeder Eingabestelle mindestens eine Marke (Token) liegt. Schalten heißt, dass aus jeder Eingabestelle der schaltenden Transition ein Token abgezogen und jeder Ausgabestelle ein Token hinzugefügt wird.

Die folgenden Grafiken zeigen ein Petrinetz mit seinen jeweiligen Zuständen nach der Durchführung der Schaltoperationen. Im Startzustand in Abb. 2.19 hat nur die Stelle
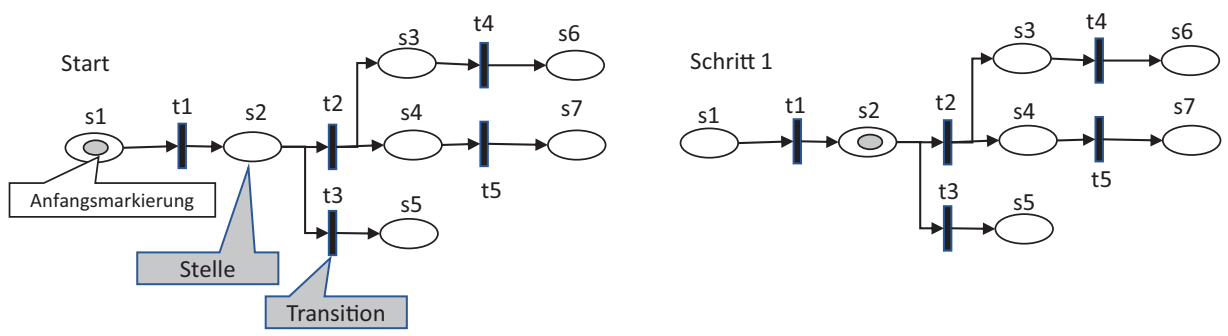

Abb. 2.19 Petrinetz I 
s1 eine Markierung, die Initial- oder Anfangsmarkierung. Die Stelle s1 ist die einzige Eingangsstelle der Transition t1, die deshalb schalten kann. Die Markierung wird aus s1 abgezogen und eine Markierung zur einzigen Ausgangsstelle s2 hinzugefügt. Die rechte Hälfte des folgenden Bildes zeigt den Zustand nach dem Schalten von $\mathrm{t} 1$.

Die Stelle s2 ist die Eingangsstelle der beiden Transitionen t2 und t3 (vgl. linke Seite von Abb. 2.20). Es könnte also die Transition $t 2$ oder $\mathrm{t} 3$ schalten, aber nicht beide. Welche Transition schaltet ist zufällig. Damit haben wir einen sogenannten nichtdeterministischen Zustand. Schaltet $\mathrm{t} 3$, wird der Stelle s5 ein Token hinzugefügt und ein Endzustand erreicht, da aus s5 keine Kante mehr herausführt. Schaltet die Transition t2, so wird den Stellen s3 und s4, die Ausgangsstellen von t2 sind, jeweils ein Token hinzugefügt. Den entsprechenden Zustand zeigt die rechte Hälfte von Abb. 2.20.

Nun kann entweder die Transition 44 oder 15 schalten. Hier könnte man folgern, dass diese beiden Transitionen gleichzeitig schalten. Dies ist aber in Petrinetzen nicht erlaubt. $\mathrm{Zu}$ einem Zeitpunkt darf nur genau eine Transition schalten. Es ist also keine echte Parallelität möglich. Es ist aber willkürlich, ob nun zuerst t4 oder t5 schaltet. In unserem Beispiel schaltet erst $\mathrm{t} 4$ und dann $\mathrm{t} 5$. Wenn beide Transitionen geschaltet haben, ist wieder ein Endzustand erreicht. Abb. 2.21 zeigt die entsprechenden Netzzustände.

Petrinetzmodelle erlauben die Analyse und Simulation von dynamischen Systemen mit nebenläufigen und nicht-deterministischen Vorgängen.

Bei der vorgestellten Art der Petrinetze handelt es sich um die Grundversion. Mit ihr lassen sich bestimmte Sachverhalte nicht beschreiben. Beispielsweise ist es nicht möglich, Prioritäten für Transitionen zu vergeben, weshalb z. B. die Rechts-vor-links-Regel an Verkehrskreuzungen nicht modelliert werden kann.

Um solche Defizite zu beheben wurden Erweiterungen eingeführt, mit denen man dem Modell weitere Aspekte der Wirklichkeit hinzuzufügen bzw. bestimmte Sachverhalte

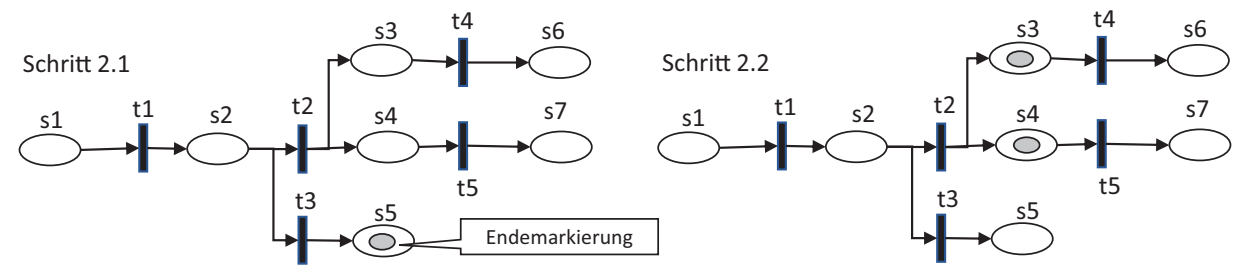

Abb. 2.20 Petrinetz II
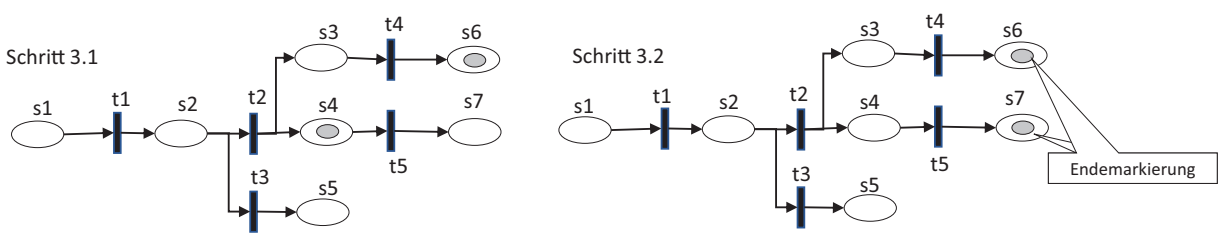

Abb. 2.21 Petrinetz III 
kompakter zu beschreiben kann. Beispiele sind mehrwertige, bunte oder mit Prioritäten versehene Petrinetze ${ }^{6}$.

\subsubsection{Calculus of Communicating Systems}

Der Calculus of Communicating Systems (CCS) wurde von Robin Milner im Jahr 1980 [24] publiziert. Dieser Kalkül erlaubt die formale Modellierung von parallelen kommunizierenden Systemen. Damit können vernetzte Systeme mit einer statischen Topologie beschrieben werden. Mit CCS können Eigenschaften von Programmen wie Verklemmungen, Verhaltensgleichheit etc. formal untersucht werden. CCS erlaubt die Beschreibung von folgender Aspekte:

- Kommunikation zwischen Akteuren über Kanäle

- Interaktion mit der Umgebung, d. h. Reaktivität

- Parallele Komposition

- Verbergen von Aktionen gegenüber der Umwelt (Information Hiding)

- Nicht-deterministische Verzweigungen

Der Ablauf eines Prozesses wird als Baum beschrieben, d. h. es gibt eine Wurzel, die den Anfangszustand darstellt und von der die einzelnen Äste ausgehen. Jeder der Äste ist markiert. Diese Markierungen repräsentieren die Aktionen, die ausgeführt werden, um von einem Zustand zum Folgezustand zu gelangen. Es wird zwischen beobachtbaren und nicht beobachtbaren Aktionen unterschieden. Nicht beobachtbare Aktionen können innerhalb eines Prozesses jederzeit ausgeführt werden, ohne dass dies Auswirkungen auf andere Prozesse hat. Prozesse haben keine gemeinsamen Variablen.

Um das Verhalten eines Prozesses zu beschreiben werden rekursive Ausdrücke verwendet. Innerhalb von Verhaltensausdrücken können Variablen verwendet werden, um andere Verhaltensausdrücke zu referenzieren.Die Verhaltensausdrücke werden gemäß folgender Syntax beschrieben, bei der Großbuchstaben Prozessnamen und Kleinbuchstaben Aktionen bezeichnen.

- Leerer Prozess:

$\varnothing$

- Aktion

Der Prozess a.P1 führt die Aktion a aus und verhält sich dann wie P1

- Prozessname

Mit dem Ausdruck A: = P1 erhält der Prozess P1 den Namen A. Da rekursive Definitionen erlaubt sind kann in dem Ausdruck P1 der Name A wieder enthalten sein.

${ }^{6}$ Einzelheiten zu solchen Erweiterungen finden sich in der verfügbaren Literatur, wie z. B. bei [23]. 
- Auswahl

Der Prozess P1 + P2 kann entweder mit dem Prozess P1 oder dem Prozess P2 fortgesetzt werden.

- Parallelkomposition

P1|P2 bedeutet, dass die Prozesse P1 und P2 parallel ausgeführt werden

- Umbenennung

$\mathrm{P} 1[\mathrm{~b} / \mathrm{a}]$ beschreibt den Prozess P1, in dem alle Aktionen mit der Bezeichnung a in $\mathrm{b}$ umbenannt werden.

- Restriktion

$\mathrm{P} 1 \backslash$ a bezeichnet den Prozess P1 ohne die Aktion a

Zueinander passende Ein- und Ausgabeaktionen in zwei unterschiedlichen Prozessen können sich synchronisieren und zu einer internen Aktion $\tau$ werden. Im Allgemeinen wird die Koaktion zu einer Aktion mit einem Querstrich über dem Aktionsnamen gekennzeichnet. Diese komplementären Aktionen sind die Sende- und Empfangsaktionen. Abb. 2.22 zeigt beispielhaft die Interaktion zwischen zwei Prozessen.

Das folgende Beispiel (siehe Abb. 2.23) zeigt, wie mit CCS ein einfacher Urlaubsantragsprozess beschrieben werden kann.

Im Prozess Mitarbeiter wird die Sendeaktion Urlaubsantrag ausgeführt (Aktionsname mit Überstrich). Danach wird auf die Nachrichten , abgelehnt ‘ oder , genehmigt ${ }^{\text {* }}$ gewartet. Der Manager empfängt die Nachricht Urlaubsantrag, die er entweder mit der

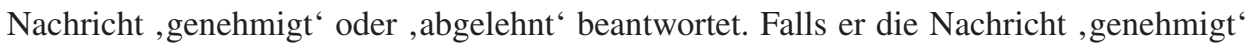
sendet, wird danach auch noch die Nachricht, genehmigter Urlaubsantrag ' gesendet. Diese Nachricht empfängt die Reisestelle. Der Austausch von Nachrichten erfolgt in CCS asynchron, d. h. ein Sender wartet so lange, bis der Empfänger die dazugehörige Empfangsaktion ausführt.

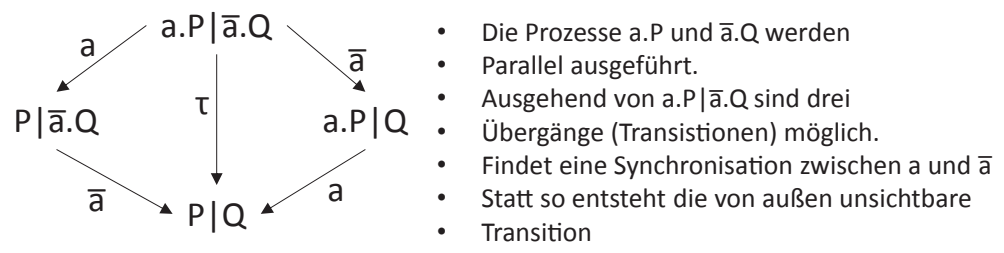

Abb. 2.22 Prozessinteraktion mit CCS

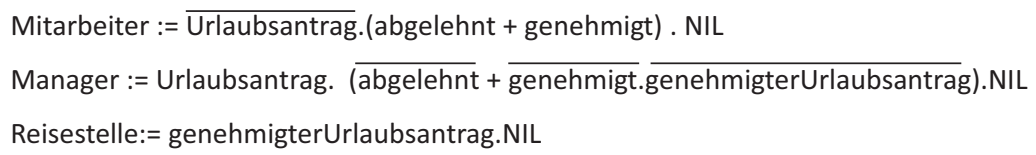

Urlaubsantragsprozess := Mitarbeiter | Manager | Reisestelle

Abb. 2.23 Beispielprozess in CCS 
$\mathrm{Zu}$ der ausgeführten informellen Interpretation gibt es auch formale Ableitungsregeln. Damit werden Prozessdefinitionen zugänglich für formale Untersuchungen.

\subsection{6 $\pi$-Kalkül}

CCS erlaubt nur statische Prozessstrukturen. Kommunikationsbeziehungen können nicht dynamisch verändert werden. Der $\pi$-Kalkül [25], ebenfalls von Robin Milner entwickelt, erlaubt die Darstellung von Prozessen mit wechselnden Strukturen. Es sind beliebige Verbindungen zwischen Komponenten darstellbar und diese Verknüpfungen können sich auch ändern bzw. neue können entstehen. Damit ist der $\pi$-Kalkül eine Erweiterung des CCS um Nebenläufigkeit. Die Notation im $\pi$-Kalkül orientiert sich weitgehend an der CCS-Notation. Das folgende Beispiel erläutert die Modellierungsmöglichkeiten des $\pi$-Kalküls (vgl. Abb. 2.24).

Der Agent (Prozess) P möchte den Wert 7 an Q über einen Link a senden. Allerdings soll der Wert indirekt über einen weiteren Agenten R übertragen werden.

Die einzelnen Schritte bei der Ausführung des Systems O sind in Abb. 2.25 sichtbar.

Die Prozesse P, R und Q werden parallel ausgeführt. Prozess P sendet über den Kanal b den Namen a und dann den Namen 7. Der Prozess P erhält über den Kanal b die beiden Namen. Dies bedeutet, alle x werden durch a ersetzt und alle z durch 7. In Abb. 2.25 ist dies das Ergebnis nach Schritt 2. Nun kann über den Kanal a der Name 7 gesendet werden, der vom Prozess Q dann angenommen wird. Damit wurde der Wert 7 über den Prozess R an den Prozess Q gesendet. Die Abb. 2.26 zeigt, wie durch den Ablauf des Systems O seine Struktur geändert wird.

Da der Kanalname a von P nach R gesendet wird, sind nach der Annahme der Nachricht die Prozesse R und Q über den Kanal a verknüpft. Damit wird deutlich, dass der $\pi$-Kalkül im Gegensatz zu CCS die Modellierung von Strukturänderungen zulässt.

- $\mathrm{P}:=\overline{\mathrm{ba}} \cdot \overline{\mathrm{b}} \overline{7} \cdot \mathrm{P}^{\prime} \quad$ Prozess $\mathrm{P}$ sendet über den Kanal b den Wert a und nachfolgend den Wert 7 und verhält sich dann wie P'.

- $\quad \mathrm{R}:=\mathrm{b}(\mathrm{x}) \cdot \mathrm{b}(\mathrm{z}) \cdot \overline{\mathrm{x} z} .0$ Prozess $\mathrm{R}$ empfängt über den Kanal $\mathrm{b}$ jeglichen Wert. Dies bedeutet in $\mathrm{R}$ wird $\mathrm{x}$ und $\mathrm{z}$ durch die empfangenen Werte ersetzt.

- $\mathrm{Q}:=\mathrm{a}(\mathrm{x}) \cdot \mathrm{Q} \quad$ Prozess $\mathrm{Q}$ empfängt über den Kanal a einen beliebigen Wert für $\mathrm{x}$. Der empfangene Wert wird überall dort eingesetzt wo sich $x$ befindet.

\section{- O:=P IRIQ Gesamtsystem O}

Abb. 2.24 Prozessbeschreibung mit $\pi$-Kalkül 
Abb. 2.25 Schritte bei der Ausführung des Systems O
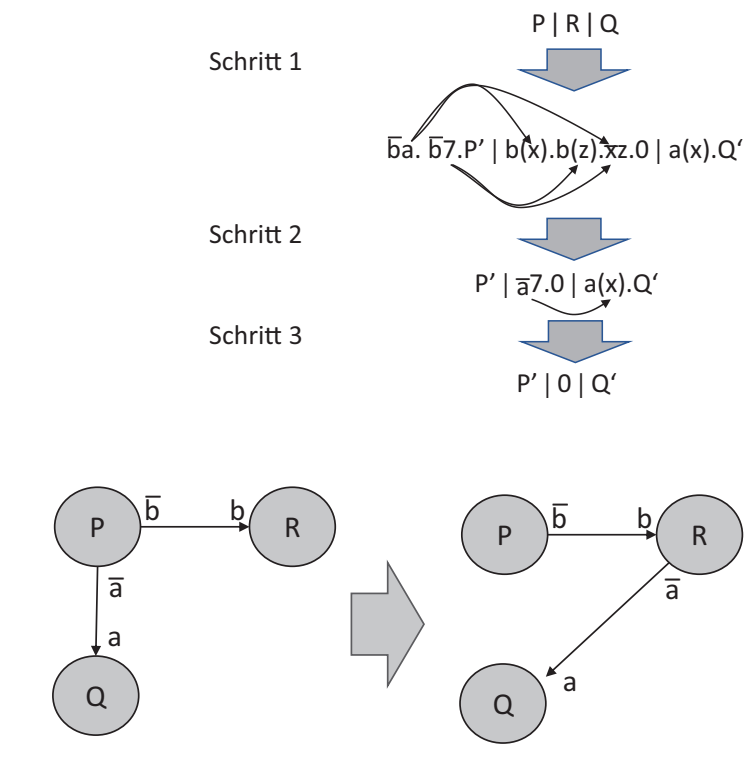

Abb. 2.26 Strukturänderung

\subsubsection{Communicating Sequential Processes}

Communicating Sequential Processes (CSP) ist eine Methodik zur Beschreibung von Interaktion zwischen kommunizierenden Prozessen. Die Idee hat Tony Hoare erstmals 1978 als imperative Sprache vorgestellt [26], dann zu einer formalen Algebra ausgebaut und 1985 mit der Veröffentlichung des Buchs mit dem Titel Communicating Sequential Processes berühmt gemacht [27].

In CSP ist wie in CCS die Anzahl der Prozesse statisch. Sie kann während der Laufzeit nicht geändert werden und zwischen Prozessen gibt es keine gemeinsamen Variablen. Stattdessen ,kennen“ sich die Prozesse und verständigen sich untereinander durch das Senden und Empfangen von Nachrichten. Zum Senden führt der Sendeprozess P das Ausgabekommando.

Q! (expr)

und der Empfängerprozess Q das Eingabekommando

P ? (vars)

aus. Ausgabe- und Eingabekommandos heißen korrespondierend, falls die Folge von Ausdrücken (expr) und die Folge von Variablen (vars) in der Anzahl und komponentenweise vom Typ übereinstimmen. Analog CCS und dem $\pi$-Kalkül basiert CSP auf einem ungepufferten Nachrichtenaustausch, bei dem der Sende- und der Empfangsprozess explizit genannt werden müssen.

Mit Q ! () und P ? () wird eine Nachricht ohne Inhalt gesendet. Solche Nachrichten werden als Signale bezeichnet und dienen einzig und allein der Synchronisation von Prozessen. Werden unterschiedliche Signale benötigt, so wird die Unterscheidung durch Typbezeichner der Form Q ! (Signal1) und P ? (Signal1) ausgedrückt. 
Neben dem oben beschriebenen unbedingten Nachrichtenaustausch gibt es die Empfangsanweisung innerhalb eines sogenannten Guarded Commands (Bewachte Anweisung). Eine bewachte Anweisung wird nur ausgeführt, wenn die vorangestellte Bool'sche Bedingung wahr ist. So wird der Formelsatz.

$$
\mathrm{x}>\mathrm{y} ; \mathrm{P} ?(\mathrm{z})->\mathrm{x}:=\mathrm{x}+\mathrm{y} ; \mathrm{y}:=\mathrm{z}
$$

nur ausgeführt, wenn $\mathrm{x}$ größer als $\mathrm{y}$ ist. Dann wird die Nachricht von $\mathrm{P}$ entgegengenommen, falls $\mathrm{P}$ bereit ist, die Nachricht zu senden.

Um auf Nachrichten von unterschiedlichen Sendern warten zu können, werden mehrere Guarded Commands zu einer Alternativanweisung zusammengeschlossen.

$$
[\mathrm{x}>\mathrm{y} ; \mathrm{P} ?(\mathrm{z})->\mathrm{x}:=\mathrm{x}+\mathrm{y} ; \mathrm{y}:=\mathrm{z} \| \mathrm{x}<\mathrm{y} ; \mathrm{Q} ?(\mathrm{z})->\mathrm{y}:=\mathrm{x}+\mathrm{y} ; \mathrm{y}:=\mathrm{z}]
$$

Im Fall $x>Y$ wird die Nachricht P?(z) erwartet, im Fall $x<y$ die Nachricht Q?(z). Alternativanweisungen können auch wiederholt ausgeführt werden. Syntaktisch wird dies durch einen der Alternativanweisung vorangestellten * ausgedrückt. Die Anweisung.

$*[\mathrm{x}>\mathrm{y} ; \mathrm{P} ?(\mathrm{z})->\mathrm{x}:=\mathrm{x}+\mathrm{y} ; \mathrm{y}:=\mathrm{z} \| \mathrm{x}<\mathrm{y} ; \mathrm{Q} ?(\mathrm{z})->\mathrm{y}:=\mathrm{x}+\mathrm{y} ; \mathrm{y}:=\mathrm{z}]$ wird solange ausgeführt, bis keine der Bedingungen mehr wahr ist - dann wird die Wiederholung beendet.

Die Konzepte bezüglich der Nebenläufigkeit von CSP dienen als Gestaltungsgrundlage der Programmiersprache Go.

\subsubsection{Abstract State Machines}

Eine abstrakte Zustandsmaschine (Abstract State Machine (ASM)) ist in der Informatik ein Modell zur formalen, operationellen Beschreibung von Algorithmen. Die Zustände einer abstrakten Zustandsmaschine sind allgemeine mathematische Strukturen. Der Erfinder des Modells ist Yuri Gurevich. Egon Börger hat die ASM für die praktische Anwendung weiterentwickelt [28].

Abstract State Machines (ASM) sind endliche Mengen von Transitionsregeln der Form.

If condition then action

mit denen die Zustände einer ASM geändert werden. Condition ist dabei ein beliebiger logischer Ausdruck und Action eine beliebige Handlung. In der Regel ist Action eine Wertzuweisung der Form $\mathrm{f}(\mathrm{t} 1, \ldots \ldots . \mathrm{tn}):=\mathrm{s}$. Die Bedeutung der Regel besteht darin, im gegenwärtigen Zustand die angegebene Regel auszuführen, wenn die angegebene Bedingung in diesem Zustand erfüllt ist. ASM-Zustände werden allgemein als beliebige Mengen beliebiger Elemente mit beliebigen darauf definierten Funktionen (Operationen) und Prädikaten definiert. Im Fall von Geschäftsobjekten sind die Elemente Platzhalter für Werte beliebigen Typs und Operationen wie Erzeugen, Duplizieren, Löschen oder algebraische Manipulationen von Objekten. 
Ein Berechnungsschritt einer ASM in einem bestimmten Zustand bedeutet, dass alle Actions, für die die Bedingung (Condition) wahr ist, gleichzeitig ausgeführt werden. Durch die simultane Ausführung kann von irrelevanten Reihenfolgen abstrahiert werden.

Mehrere ASMs können gleichzeitig ablaufen und über sogenannte Controlled bzw. Monitored Functions verknüpft werden. Eine gegebene ASM M kann Controlled Functions aktualisieren, sie kann aber nicht von anderen ASM aus der Umgebung von M verändert werden. Monitored Functions einer gegebenen ASM M können nur durch deren Umgebung aktualisiert werden. Durch die paarweise Verwendung von Controlled and Monitored Functions kann ein Netzwerk paralleler sich koordinierender ASMs aufgebaut werden.

\subsubsection{Objektorientierte Modelle}

Die bisher betrachteten Modelle der Informatik legen den Schwerpunkt entweder auf Daten oder Funktionen. Die Darstellung von Daten in einem ERM und Funktionen in einem Flussdiagramm ergänzen sich nur in entkoppelten Darstellungen - die Unterscheidung in Daten- und Funktionssicht bei ARIS macht dies deutlich. Die objektorientierte Modellbildung reduziert diese isolierten Sachverhalte nicht mehr auf ihre Einzelteile, sondern betrachtet sie als integriertes Ganzes, bei dem die einzelnen Komponenten miteinander verbunden und voneinander abhängig sind.

Unter einem objektorientierten Modell versteht man eine Sichtweise auf ein komplexes System, bei der dieses durch das Zusammenspiel von Objekten beschrieben wird. Durch diese Art der Modellbildung soll die Komplexität der Beschreibung für Sachverhalte, die in Software abzubilden sind, reduziert werden. Die Objektorientierung sieht die in der realen Welt vorkommenden Entitäten als Objekte. Ein Telefon ist ebenso ein Objekt, wie ein Fahrrad, ein Mensch oder ein Mitarbeiter. Solche Objekte setzen sich wiederum aus anderen Objekten zusammen wie z. B. Schrauben, Stangen, Armen, Füßen, Kopf o. Ä. Die Objekte werden, wie in der Modellbildung üblich, auf ihre in der jeweiligen Situation bedeutsamen Eigenschaften reduziert. So wird z. B. ein Mitarbeiter in einem Lohnabrechnungssystem auf Name, Adresse, Mitarbeiternummer, vereinbartes Einkommen, Steuerklasse usw. reduziert.

Die in einem Modell berücksichtigten Objekte werden nicht einzeln konzipiert. Für gleichartige Objekte wird ein grober Bauplan mit gleichartigen Eigenschaften erstellt. Beispielsweise modelliert man für eine Bibliotheksanwendung die Eigenschaften von Büchern, die für allen Büchern identisch sind. Solche allgemeinen Beschreibungen von Objekten werden in der Objektorientierung Klassen genannt. Mit diesen Klassen werden dann innerhalb des Modells die benötigten konkreten Objekte erzeugt (Instanzen).

Die Abbildung der Wirklichkeit in ein Modell erfolgt also zweistufig. Zuerst werden gleichartige Objekte der Wirklichkeit identifiziert und jeweils als Klasse beschrieben. Die einzelnen Objekte werden dann als sogenannte Instanzen einer Klasse erzeugt. Eine Klasse beschreibt somit die Struktur einer Menge gleichartiger Objekte. Abb. 2.27 zeigt eine Klasse-Objekt Beziehung, nach der das Buch „Subjektorientiertes 


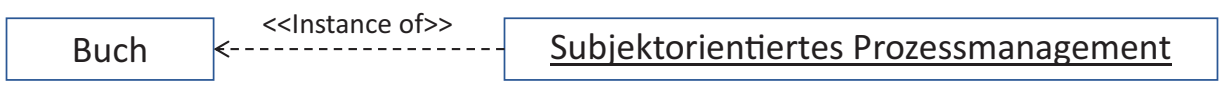

Abb. 2.27 Beziehung Klasse-Objekt

Prozessmanagement“ eine Instanz der Klasse Buch ist. Die verwendete Notation entstammt der Unified Modeling Language (UML). UML ist eine von der Object Management Group standardisierte Sprache zur Beschreibung objektorientierter Modelle.

Die Eigenschaften einer Klasse sind

- ihre Bestandteile und die in ihnen enthaltenen Daten und Informationen, auch Attribute genannt,

- die auf den Bestandteilen definierten Operationen und ihre Parameter (Methoden), mit denen ein Objekt manipuliert bzw. dessen Zustand abgefragt werden kann und

- Bedingungen, Voraussetzungen und Regeln, die die Objekte erfüllen müssen (Zusicherungen).

Abb. 2.28 zeigt ein Beispiel für die Beschreibung der Klasse Buch. Diese Klasse hat fünf Attribute. Das Attribut Seitenzahl hat eine Zusicherung, die besagt, dass die Seitenzahl positiv sein muss. Die Klasse erlaubt den Zugriff und die Manipulation ihrer Daten mit sechs Methoden. So können der Titel, die Autoren, die Seitenzahl, der Verlag und der Inhalt gesetzt werden (Set). Mit der Operation (Methode) AnzeigenSeite(Seite) kann der Inhalt der angegebenen Seite aus dem Buch ,,ausgelesen“ werden.

Ausgehend von einer solchen Klassendefinition können nun Objekte instanziiert werden. Mithilfe der Operationen können dann für jede dieser Instanzen die jeweiligen Attribute entsprechend gesetzt werden. Ein objektorientiertes Modell beschreibt neben den Definitionen der Klassen und der zugehörigen Objekte auch die Beziehungen der Klassen zueinander. Die Arten von Beziehungen sind:

- Vererbung

Eigenschaften können von einer Klasse zur nächsten weitergegeben werden. Man spricht dann von Vererbung. Die Klasse, die vererbt, heißt Oberklasse, und die Klasse, die erbt, Unterklasse. So ist eine Klasse Notizbuch Unterklasse der Klasse Buch. Der Klasse Notizbuch wird noch die Methode „Seiteneintrag (Seite, Inhalt)“ hinzugefügt. Damit kann auf der angegebenen Seite ein Text eingegeben werden; Ansonsten erbt die Unterklasse Buch alle Attribute und Methoden von der Klasse Buch. Abb. 2.29 zeigt die Vererbungsbeziehung zwischen Buch und Notizbuch. Der Dreieckspfeil zeigt von der Unterklasse zur Oberklasse.

- Assoziationen

Eine Assoziation ist eine Beziehung zwischen verschiedenen Objekten einer oder mehrerer Klassen. Dargestellt werden Assoziationen als einfache Linie zwischen zwei Klassen. Die Linie kann mit einem Namen (Bezeichnung) und Anzahlangaben versehen werden. Auch die assoziierten Klassen können Namen bezüglich der Beziehung 
Abb. 2.28 Beschreibung der Klasse Buch

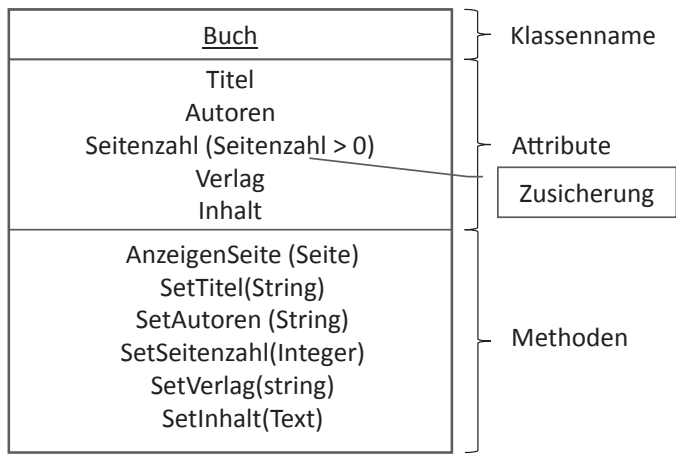

Abb. 2.29 Vererbung von Eigenschaften

Abb. 2.30 Assoziationen zwischen Objekten

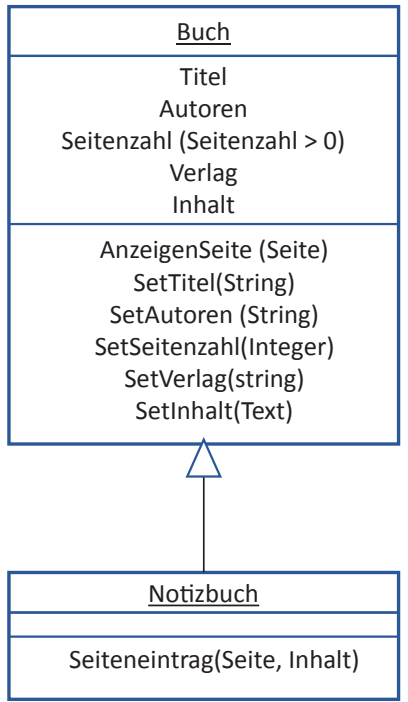

\begin{tabular}{|c|c|c|c|c|}
\hline Firma & $0 . .1$ & beschäftigt> & $0 .{ }^{*}$ & Persor \\
\hline
\end{tabular}

erhalten. Abb. 2.30 illustriert ein Beispiel für eine Assoziation. Eine Person (Arbeitnehmer) kann bei keiner oder einer Firma (Arbeitgeber) beschäftigt sein und eine Firma kann keine oder mehrere Personen beschäftigen. Assoziationen haben eine große Ähnlichkeit mit den Entity-Relationship-Diagrammen.

- Aggregationen

Aggregationen sind eine Variante von Assoziationen. Dabei handelt es sich ebenfalls um eine Beziehung zwischen zwei Klassen, jedoch mit der Besonderheit, dass die zwei Klassen zueinander in Beziehung stehen wie ein Teil zu einem Ganzen. Eine Aggregation setzt sich zusammen aus einer Menge von Einzelteilen. Abb. 2.31 zeigt ein Aggregationsbeispiel, bei dem ein Unternehmen aus Abteilungen, und eine Abteilung aus Mitarbeitern besteht. 


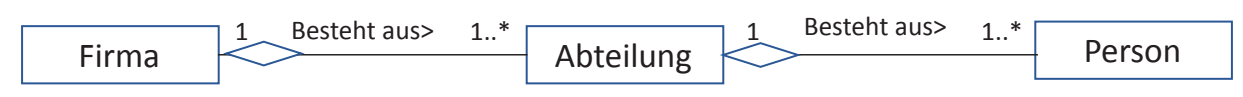

Abb. 2.31 Mehrstufige Aggregation

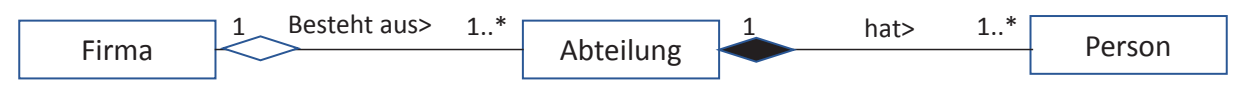

Abb. 2.32 Komposition

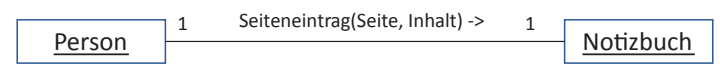

Abb. 2.33 Nachricht zwischen Objekten

- Komposition

Eine besondere Form der Aggregation ist die Komposition. Hier hängt das Ganze von der Existenz seiner Einzelteile ab. In Abb. 2.32 ist eine Änderung an der obigen Aggregation zu sehen. Eine Abteilung besteht nicht mehr, wenn ihr niemand angehört.

- Objekte kommunizieren miteinander, d. h. ein Objekt sendet Nachrichten an ein anderes Objekt. Die Nachrichten stoßen dann die zugehörigen Operationen an. Ein Objekt versteht also nur die Nachrichten, zu denen es auch die zugehörigen Operationen enthält. Abb. 2.33 zeigt die Kommunikation einer Person mit dem Notizbuch zum Eintragen einer neuen Notiz.

Die beschriebenen Konstrukte bilden den Nukleus des objektorientierten Modellierungsansatzes. Darüber hinaus existieren zahlreiche Erweiterungen zur Abbildung bestimmter Sachverhalte wie die sogenannten Behälterklassen ${ }^{7}$.

\subsubsection{Agenten-/Aktoren-orientierte Modelle}

Bislang gibt es keine standardisierte und allgemein akzeptierte Definition des Agentenkonzepts. Der Begriff wird abhängig von der Anwendungsdomäne im Detail unterschiedlich definiert [30]. All diesen Definitionen ist aber gemeinsam, dass ein Agent eine abgrenzbare Einheit ist, die in der Lage ist, die ihr vorgegebenen Aufgaben flexibel, interaktiv und autonom zu verfolgen. Oft wird der Begriff Aktor als synonym für den Begriff Agent benutzt. So definieren Lankhorst et al. [31] einen Business Actor als „,... an entity

${ }^{7}$ Weiterführende Informationen finden sich beispielsweise bei [29]. 
that is capable of performing behavior". Multiagentensysteme bestehen aus mehreren Agenten, die synchron oder asynchron Nachrichten austauschen. Multiagentensysteme können die Strukturen von Softwaresystemen abbilden oder auch der Modellbildung sozialer Systeme dienen. Je nach Anwendungssituation kann dann zwischen Softwareagenten und menschlichen Agenten unterschieden werden.

In gewisser Weise können die Prozesse in CCS, im $\pi$-Kalkül und in CSP als Multiagentensysteme betrachtet werden. Der Begriff Prozess wird dort analog zu den Begriffen Agent und Aktor verwendet.

Ein agentenorientiertes Modell beinhaltet also die Agenten, die Kommunikationspfade und die Nachrichten, die ausgetauscht werden. ${ }^{8}$

\subsection{Fazit: Modelle für Geschäftsprozesse}

Geschäftsprozessmodelle bilden den Wirklichkeitsausschnitt der Geschäftsprozesse ab. Das subjektive Verständnis des Geschäftsprozessbegriffs beeinflusst, welche Prozessaspekte als wesentlich erachtet werden und deshalb in den Modellen im Vordergrund stehen. Hier schlagen sich Absichten und Interessen des Modellerstellers nieder. Konsequenz sind zahlreiche Auslegungen des Geschäftsprozessbegriffs, von denen jede weder richtig noch falsch ist, sondern lediglich andere Betrachtungsschwerpunkte setzt.Als Beispiele dafür können folgende Definitionen des Terminus Geschäftsprozess gelten:

1. „Folge von Wertschöpfungsaktivitäten (Wertschöpfung) mit einem oder mehreren Inputs und einem Kundennutzen stiftenden Output“" [33].

2. „Ein Prozess ist die inhaltlich abgeschlossene, zeitliche und sachlogische Folge von Aktivitäten, die zur Bearbeitung eines betriebswirtschaftlich relevanten Objekts notwendig sind.“[34].

Beide Definitionen stellen auf die erforderlichen Aktivitäten und deren Folgen ab. Das erste Beispiel erwähnt zusätzlich den Input und den Output mit dem Kundennutzen, während in der zweiten Definition stattdessen die Bearbeitung der Geschäftsobjekte einbezogen wird.

Handelnde und benötigte Ressourcen fehlen dagegen in beiden Begriffsfassungen. Sie betrachten nicht, durch wen und womit die Aktivitäten ausgeführt werden. Es gibt keinen Bezug zur Organisation, in die ein Geschäftsprozess eingebettet ist, sowie dazu, welche IT-Anwendungen oder sonstige Ressourcen eingesetzt werden, um ihn auszuführen.Wir

${ }^{8}$ Eine Sammlung von agentenorientierten Modellierungssprachen und den dazugehörigen Vorgehensweisen findet sich in [32]. 
folgen deshalb in Anlehnung an Gerhard Schewe einem Begriffsverständnis, das auch diese fehlenden Aspekte berücksichtigt [33]:

1. Ein Prozess ist die Summe von miteinander verknüpften Aktivitäten (Aufgaben),

2. die von Handelnden (Menschen, Systeme als Aufgabenträger)

3. in sachlogischer und zeitlicher Reihenfolge

4. mit Hilfsmitteln (Sachmittel, Information)

5. zur Bearbeitung eines Geschäftsobjekts ausgeführt werden,

6. um ein Kundenbedürfnis zu befriedigen (und damit zur Wertschöpfung beizutragen),

7. und einen definierten Anfang und Input

8. sowie ein definiertes Ende und Ergebnis aufweisen.

Wie bereits in Abschn. 1.3 ausgeführt, formen wir die Bestandteile etwas um und gruppieren sie wie folgt:

9. Prozessstrategie: Ein Prozess hat

a) einen definierten Anfang und Input (Startereignis),

b) und weist ein definiertes Ende mit einem Ergebnis auf,

c) das zur Befriedigung eines Kundenbedürfnisses (und damit zur Wertschöpfung) beiträgt

10. Prozesslogik: Ein Prozess

a) ist die Summe von miteinander verknüpften Aktivitäten (Aufgaben),

b) die nach dem Startereignis von Handelnden

c) in sachlogischer und zeitlicher Reihenfolge

d) zur Bearbeitung eines Geschäftsobjekts ausgeführt werden um

e) das gewünschte Ergebnis zu erzeugen.

11. Prozessrealisierung: Ein Prozess wird realisiert

a) mit Menschen und/oder Maschinen, die Aufgaben der jeweiligen Handelnden übernehmen, und diese

b) mit Hilfsmitteln (Sachmittel, Information, Anwendungsprogramme etc.) ausführen.

Mit diesem Verständnis von Geschäftsprozessen wird der Bezug der in diesem Kapitel beschriebenen Modelle aus unterschiedlichen Domänen zum Geschäftsprozessmanagement deutlich. Abb. 2.34 zeigt entsprechend den integrativen Charakter der Geschäftsprozessmodelle.Die Modelle von Habermas und Luhmann befassen sich mit Aspekten der gesellschaftlichen Systeme und Organisationen. Sie beschreiben welche Komponenten und Beziehungen eine Organisation ausmachen und wie Menschen darin positioniert sind. Komplexe Organisationen können beispielsweise anhand betrieblicher Funktionen, Leistungsspektrum, geografischer Aspekten oder Kombinationen davon in Teilorganisationen strukturiert werden. Das Ergebnis ist das Organigramm. 


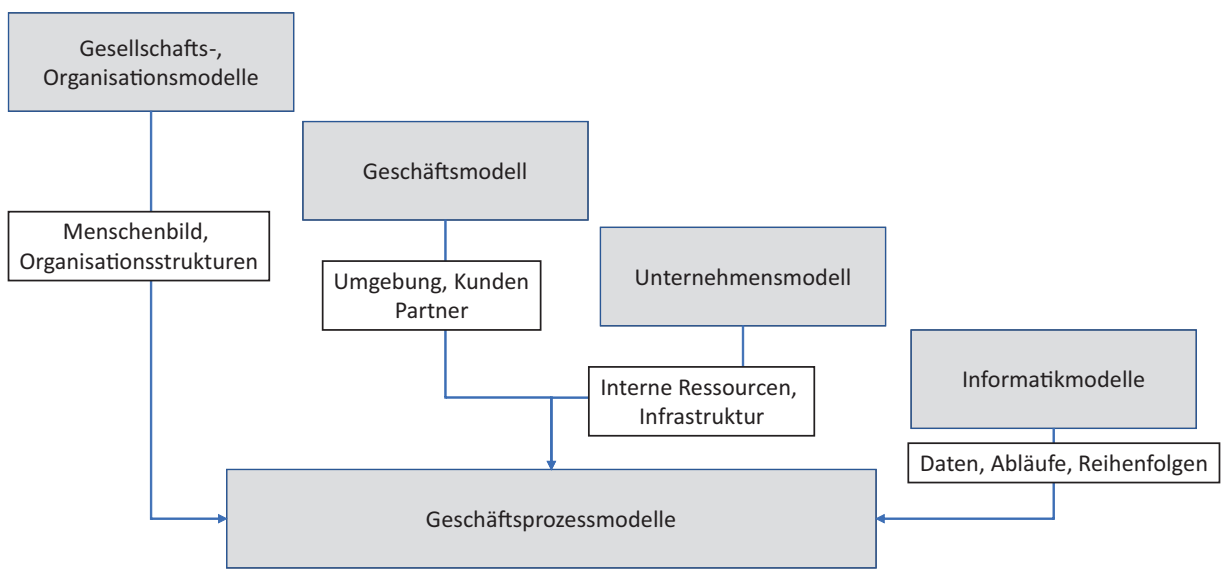

Abb. 2.34 Integration verschiedener Modelle durch Geschäftsprozessmodelle

Geschäftsmodelle betrachten unter anderem die Aspekte Kunden, Lieferanten, Partner und Wertschöpfung und blicken damit zunächst auf die externen Leistungsbeziehungen. Insbesondere mit Wertversprechen (Produkte und Services), Aktivitäten und Ressourcen stellen sie aber auch die Verbindung zur stärker nach innen gerichteten Unternehmensarchitektur her. In dieser sind auf der fachlichen Ebene die Aufbauorganisation mit den personellen Ressourcen, die Prozesse und die logischen Geschäftsobjekte modelliert. Die Verknüpfung mit der technischen Schicht der Unternehmensarchitektur führt zu den Modellen aus der Informatik. Diese beschreiben etwa Datenstrukturen, Kontrollflüsse und Algorithmen für Programme sowie die Gestalt und das Zusammenwirken weiterer informations- und kommunikationstechnischer Komponenten, die für die Ausführung gewünschter Aktionen im Rahmen der Prozessunterstützung und -automatisierung nötig sind.

\section{Literatur}

1. Markus Gabriel, Warum es die Welt nicht gibt, Ullstein Taschenbuch (7. Januar 2015)

2. O. Siemoneit; Eine Wissenschaftstheorie der Betriebswirtschaftslehre; Dissertationsschrift, Universität Stuttgart; 2010

3. Herbert Stachowiak, Allgemeine Modelltheorie, Springer Verlag, Wien New York, 1973, S. 131

4. Alfred Kieser, Peter Walgenbach; Organisation; Schäffer-Pöschl Verlag, Stuttgart 2007

5. David Crowther, Miriam Green; Organisational Theory; Chartered Institute of Personnel Development, London 2004

6. Georg Schreyögg; Organisation; Gabler Verlag, 5. Auflage, Wiesbaden 2008

7. A. Kieser, Mark Ebers; Organisationstheorien; Kohlhammer Verlag; Stuttgart 2006, S. 68 
8. Jürgen Habermas, Theorie des kommunikativen Handelns Band 1,Auflage 2014, Suhrkamp Taschenbuch Wissenschaft, Frankfurt am Main 1981, S. 128

9. Margot Berghaus; Luhmann leicht gemacht; Böhlau Verlag, 2. Auflage, Köln 2004 und Niklas Luhmann; Soziale Systeme, Suhrkamp Verlag, Frankfurt 1984

10. Christian Scheer, Thomas Deelmann, Prof. Dr. Peter Loos; Geschäftsmodelle und internetbasierte Geschäftsmodelle - Begriffsbestimmung und Teilnehmermodell; Working Papers of the Research Group Information Systems \& Management, Universität Mainz 2003

11. Bernd W. Wirtz, Oliver Schilke and Sebastian Ullrich, Strategic Development of Business Models Implications of the Web 2.0 for Creating Value on the Internet, Long Range Planning 43 (2010) 272-290, Elsevier 2010

12. Alexander Osterwalder, Yves Pigneur; Business Model Generation; John Wiley \& Sons, Hoboken 2010

13. Kaplan R.S. Norten D.P.; (1997) Balanced Scorecard; Schaeffer-Poeschl Verlag

14. Springer Gabler Verlag (Herausgeber), Gabler Wirtschaftslexikon, Stichwort: Balanced Scorecard, online im Internet: http://wirtschaftslexikon.gabler.de/Archiv/1856/balanced-scorecard-v8.html. letzter Zugriff am 22.02.2018

15. V. Allee; Value Networks and true nature of collaboration; Meghan Kiffer Press; Tampa Fl. 2015

16. Wächter H. (eds) Selbstverständnis betriebswirtschaftlicher Forschung und Lehre. Gabler Verlag, Wiesbaden

17. ITIL Best Practice; https://www.axelos.com/best-practice-solutions/itil; Last Access February 2018

18. COBIT 5; http://www.isaca.org/COBIT/Pages/COBIT-5-german.aspx. Last Access February 2018

19. Dirk Matthes; Enterprise Architecture Kompendium; Springer Verlag, Berlin Heidelberg 2011, Seite 5

20. Szyperski, N.; Informationsbedarf. In E. Grochla (Hrsg.), Handwörterbuch der Organisation, S. 904. Verlag C.E. Poeschel, Stuttgart, 2. Auflage, 1980

21. Steinmüller, W.: Eine sozialwissenschaftliche Konzeption der Informationswissenschaft. Nachrichten für Dokumentation, 23 (1981) Nr. 2, 69-80

22. P. P.-S. Chen: Entity-Relationship Modeling - Historical Events, Future Trends, and Lessons Learned (PDF-Datei; 417 kB). In: M. Broy, E. Denert (Hrsg.): Software Pioneers: Contributions to Software Engineering. Springer-Verlag, Berlin 2002.

23. Reisig W.; Petrinetze: Modellierungstechnik, Analysemethoden, Fallstudien; Vieweg+Teubner Verlag; Auflage: 2010 (15. Juli 2010)

24. Robin Milner, A Calculus of Communicating Systems; Lecture Notes in Computer Science, Springer 1980

25. Robin Milner, Communicating and Mobile Systems: the $\pi$-calculus; Cambridge University Press; 1999

26. C.A.R. Hoare, Communicating Sequential Processes, Communications of the ACM, Vol. 21, Nr. 8, 1978

27. C.A.R. Hoare, Communicating Sequential Processes, Prentice Hall, Englewood Cliffs, New Jersey, 1985

28. E. Börger, R. Stärk; Abstract State Machines; Springer Verlag 2003

29. G. Booch et al.; Object-Oriented Analysis and Design with Applications; Third Edition, Addison-Wesley: Boston MA; 2007.

30. Stan Franklin, Art Graesser; Is It an Agent, or Just a Program?: A Taxonomy for Autonomous Agents; Institute For Intelligent Systems, University of Memphis, Memphis, TN 38152, USA; see https://pdfs.semanticscholar.org/288d/7952b6648.749fcbdcedabedf8f43cf7fda52.pdf. Letzter Zugriff September 2017 
31. Marc Lankhorst et al.; Enterprise Architecture at Work, Fourth Edition; Springer Verlag, Berlin 2017

32. Massimo Cossetino, et al.; Handbook on Agent-Oriented Design Processes, Springer Verlag, Berlin, 2014 und G. Wieß, R. Jakob; Agentenorientierte Softwareentwicklung.; Springer Verlag, Berlin, 2005

33. Gerhard Schewe, Geschäftsprozess, Gabler Wirtschaftslexikon. http://wirtschaftslexikon.gabler.de/Definition/geschaeftsprozess.html\#definition. letzter Zugriff 31.7.2017

34. Jörg Becker, Geschäftsprozessmodellierung, Enzyklopädie der Wirtschaftsinformatik, Online. Lexikon, http://www.enzyklopaedie-der-wirtschaftsinformatik.de/lexikon/daten-wissen/ Informationsmanagement/Information-/index.html. letzter Zugriff 31.7.2017

Open Access Dieses Kapitel wird unter der Creative Commons Namensnennung - Nicht kommerziell - Keine Bearbeitung 4.0 International Lizenz (http://creativecommons.org/licenses/ by-nc-nd/4.0/deed.de) veröffentlicht, welche die nicht-kommerzielle Nutzung, Vervielfältigung, Verbreitung und Wiedergabe in jeglichem Medium und Format erlaubt, sofern Sie den/die ursprünglichen Autor(en) und die Quelle ordnungsgemäß nennen, einen Link zur Creative Commons Lizenz beifügen und angeben, ob Änderungen vorgenommen wurden. Die Lizenz gibt Ihnen nicht das Recht, bearbeitete oder sonst wie umgestaltete Fassungen dieses Werkes zu verbreiten oder öffentlich wiederzugeben.

Die in diesem Kapitel enthaltenen Bilder und sonstiges Drittmaterial unterliegen ebenfalls der genannten Creative Commons Lizenz, sofern sich aus der Abbildungslegende nichts anderes ergibt. Sofern das betreffende Material nicht unter der genannten Creative Commons Lizenz steht und die betreffende Handlung nicht nach gesetzlichen Vorschriften erlaubt ist, ist auch für die oben aufgeführten nicht-kommerziellen Weiterverwendungen des Materials die Einwilligung des jeweiligen Rechteinhabers einzuholen.

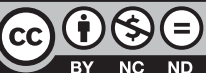

